\title{
Application of a simplified calculation for full-wave microtremor $H / V$ spectral ratio based on the diffuse field approximation to identify underground velocity structures
}

\author{
Hao $\mathrm{Wu}^{1 *}$, Kazuaki Masaki ${ }^{2}$, Kojiro Irikura ${ }^{2}$ and Francisco José Sánchez-Sesma ${ }^{3}$
}

\begin{abstract}
Under the diffuse field approximation, the full-wave (FW) microtremor $H / V$ spectral ratio $(H / V)$ is modeled as the square root of the ratio of the sum of imaginary parts of the Green's function of the horizontal components to that of the vertical one. For a given layered medium, the FW H/V can be well approximated with only surface waves (SW) $H / V$ of the "cap-layered" medium which consists of the given layered medium and a new larger velocity half-space (cap layer) at large depth. Because the contribution of surface waves can be simply obtained by the residue theorem, the computation of SW H/V of cap-layered medium is faster than that of FW HIV evaluated by discrete wavenumber method and contour integration method. The simplified computation of SW H/V was then applied to identify the underground velocity structures at six KiK-net strong-motion stations. The inverted underground velocity structures were used to evaluate FW H/Vs which were consistent with the SW H/Vs of corresponding cap-layered media. The previous study on surface waves H/Vs proposed with the distributed surface sources assumption and a fixed Rayleighto-Love waves amplitude ratio for horizontal motions showed a good agreement with the SW H/Vs of our study. The consistency between observed and theoretical spectral ratios, such as the earthquake motions of H/V spectral ratio and spectral ratio of horizontal motions between surface and bottom of borehole, indicated that the underground velocity structures identified from SW H/V of cap-layered medium were well resolved by the new method.
\end{abstract}

Keywords: Diffuse field approximation, Microtremor H/V spectral ratio, Full-wave, Surface waves, Underground velocity structures

\section{Introduction}

The microtremor $H / V$ spectral ratio $(H / V)$ is the most popular approach to estimate the predominant period of a site (Nakamura 1989, 2000; Lermo and Chávez-García 1993). In what has been controversial, Nakamura $(1989,2000)$ proposed to consider this ratio to be the empirical transfer function. Using a numerical simulation, Lachet and Bard (1994) verified that the natural period of the sedimentary site coincides well with the predominant period of $H / V$.

\footnotetext{
*Correspondence: haowu@geor.or.jp

1 Earthquake Engineering Group, Geo-Research Institute, 2-1-2, Otemae, Chuo-ku, Osaka, Japan

Full list of author information is available at the end of the article
}

These issues have raised an assortment of opinions on the characteristics and origin of features of $H / V$, i.e., predominant period and amplitude, being a $\mathrm{SH}$ waves transfer function (Nakamura 1989, 2000; Lermo and Chávez-García 1993), the role of ellipticity of fundamental mode or high modes of Rayleigh waves and the emergency of Airy phase of Love waves (Lachet and Bard 1994; Konno and Ohmachi 1998; Fäh et al. 2001; Arai and Tokimatsu 2004; BonnefoyClaudet et al. 2006a; Baan 2009). Other researchers, such as Tokimatsu and Tamura (1995), Tamura (1996), and Bonnefoy-Claudet et al. (2008), concluded that the origin is not unique, and it depends on the impedance ratio, distribution of sources (or cultural condition), and the relative proportion of body (SH, P-SV waves) and surface waves (Rayleigh 
and Love waves). Accordingly, various methods to identify underground velocity structures have been proposed for three-component station measurements, depending on different assumptions on the origin, e.g., ellipticity of fundamental mode of Rayleigh waves (Fäh et al. 2001), higher modes of surface waves (Arai and Tokimatsu 2004), or SHwave transfer function (Lin et al. 2014).

Since microtremor consists of both body and surface waves, a full-wave description is desirable to interpret the $H / V$. Diffuse field approximation is one of the promising theories to accomplish it. The diffuse field concept originated in acoustics and was firmly established for elasticity by Weaver (1982). But the roots of the idea can be traced back to the pioneering work of Aki (1957) who used ambient seismic noise (ASN) in the SPAC method (for spatial autocorrelation). The short-range cross-correlations of noise in a small array and its azimuthal average allow, through the argument of the Bessel function of zero order, to retrieve the phase velocity of Rayleigh waves. Aki's pioneering work addressed first ideas and issues that were later formalized by Weaver (1982) as diffuse fields. The connection with 2D elasticity was pointed out by SánchezSesma and Campillo (2006). It has been proposed that the coda of earthquakes can be seen as a realization of a diffuse field (Shapiro et al. 2000; Hennino et al. 2001; Margerin 2009). In several instances, the ASN allows Green's function retrieval from the average of cross-correlation between two receivers within a diffuse seismic field. Shapiro and Campillo (2004), Sabra et al. (2005), and Shapiro et al. (2005) confirmed that ASN is generally diffuse. We assert that the key of this fact lies in multiple scattering. The properties and the conditions of emergence of a diffuse field have been explored by many researchers worldwide (e.g., Weaver and Lobkis 2001; Sánchez-Sesma and Campillo 2006; Sánchez-Sesma et al. 2008). For the applications, the diffuse field assumption allows to get useful approximations of the Green's Function from cross-correlation of recorded motions. Even Mulargia (2012) who claims that ASN is not diffuse accepts that it holds significant imaging properties. Based on the diffuse field approximation, the directional energy density is proportional to the imaginary part of the Green's function when source and receiver coincide with each other both in position and direction (Sánchez-Sesma et al. 2008). The FW H/V is then expressed as the square root of the sum of imaginary parts of the horizontal components of Green's function to that of the vertical counterpart (Sánchez-Sesma et al. 2011). In the diffuse field description, the imaginary part of the Green's function incorporates naturally the contributions of both body (BW) and surface waves (SW). Therefore, an explanation on the origin of $H / V$ is achieved by full-wave.

Lontsi et al. (2015) used the FW description to invert $H / V$ recorded and processed from different depths to identify underground velocity structures at a couple of sites in Germany. They used a straightforward calculation inspired on the conventional discrete wavenumber method to evaluate the Green's functions. This full-wave approach is time consuming and limits the application of inversion of FW $H / V$ to some extent. García-Jerez et al. (2013) evaluated the imaginary parts of the Green's functions, accounting for the separate contributions of body and surface waves using the Cauchy's contour integration method, for which the computation of surface waves corresponding to residues is fast. They further developed two faster codes for inversion of both the FW $H / V$ and joint inversion, adding the velocity dispersion curve of fundamental mode of Rayleigh waves, and then implemented these codes to identify underground velocity structures at some sites (García-Jerez et al. 2016; Piña-Flores et al. 2017). That has been a major accomplishment, but the computation of body waves which contribute little to the full-wave consumes most of the computing time. Also, the efficiency of their code (opened to public) for forward calculation of FW $H / V$ reduces significantly, when the number of frequencies of $H / V$ is larger than 1000 and the number of layers is more than 5 . To circumvent those drawbacks, we explore other ways in which we see room for improvement. Harvey (1981) applied the locked-mode approximation method to approximate the Green's functions in time domain for full-wave with superposition of normal modes (surface waves) by adding a large velocity half-space to the bottom of given velocity structures. He referred to this large velocity half-space as "cap layer." Because the computation of surface waves is quite fast and the computation of body waves is not needed, the computational efficiency is better than the one achieved with other methods, such as reflectivity, ThomsonHaskell propagation matrix, and global matrix, all within the discrete wavenumber summation scheme.

In this study, we adopt the diffuse field approximation (DFA) and investigate the applicability of the cap-layered medium to compute the $H / V$ from the surface waves of that waveguide. Our approach allows identifying the underground velocity structures between the surface and bottom of boreholes at six KiK-net stations where the PS logging data are not reliable enough to explain the observed $H / V$ s. In the framework of SW $H / V$, we investigate the differences between $\mathrm{SW} H / V$ of our approach and SW $H / V$ of the previous study on microtremor $H / V$ spectral ratio developed by Arai and Tokimatsu (2004). We finally discuss the validity of identified underground velocity structures by comparing observed results with theoretical results, such as earthquake motions $H / V$ spectral ratio developed by Kawase et al. (2011) and spectral ratio of horizontal motions between surface and bottom of borehole. 


\section{Formula of microtremor $H / V$ spectral ratio based on diffuse field approximation}

Based on the diffuse field approximation, the FW $H / V$ is expressed as follows:

$$
\left(\frac{H}{V}\right)^{\mathrm{FW}}=\sqrt{\frac{\operatorname{Im} G_{11}+\operatorname{Im} G_{22}}{\operatorname{Im} G_{33}}},
$$

where $\operatorname{Im} G$ is the imaginary part of the Green's function when both source and receiver coincide with each other both in position and direction (Sánchez-Sesma et al. 2011), and the subscripts 11 and 22 mean two horizontal components, and 33 the vertical one. The imaginary part of the Green's functions "detects" energies that are both radiated from and coming back to the source. $\operatorname{Im} G_{11}$, Im $G_{22}$, and $\operatorname{Im} G_{33}$ are proportional to directional energy densities associated with the three degrees of freedom. Other components, such as $G_{12}$ (or $G_{21}$ ), $G_{13}$ (or $G_{31}$ ), and $G_{23}$ (or $G_{32}$ ), are not needed, and the imaginary parts of them are zero for coincident source and receiver. The right side of Eq. (1) only depends on the underground velocity structure, such as density, P- and S-wave velocity, and thickness of each layer, regardless of sources distribution and proportion of body and surface waves, which makes it the signature of the site. The corresponding code (Sánchez-Sesma et al. 2011) was inspired on the discrete wavenumber method to carry out the integrations in the wavenumber domain.

García-Jerez et al. (2013) proposed to evaluate the Green's functions by the Cauchy's contour integration method, because the Green's functions for surface waves can be quickly evaluated as the sum of residues. For horizontally layered media, the imaginary part of the Green's functions and the SW $H / V$ is expressed as follows:

$$
\begin{gathered}
\operatorname{Im} G_{11}^{\mathrm{SW}}=\operatorname{Im} G_{22}^{\mathrm{SW}}=-\frac{1}{4} \sum_{m=0}^{M}\left[A_{\mathrm{R} m}(u / w)_{m}^{2}\right]-\frac{1}{4} \sum_{n=0}^{N} A_{\mathrm{L} n}, \\
\operatorname{Im} G_{33}^{\mathrm{SW}}=-\frac{1}{2} \sum_{m=0}^{M} A_{\mathrm{R} m}, \\
\left(\frac{H}{V}\right)^{\mathrm{SW}}=\sqrt{\frac{\sum_{m=0}^{M}\left[A_{\mathrm{R} m}(u / w)_{m}^{2}\right]+\sum_{n=0}^{N} A_{\mathrm{L} n}}{\sum_{m=0}^{M} A_{\mathrm{R} m}}}
\end{gathered}
$$

where $A_{\mathrm{R}}$ and $A_{\mathrm{L}}$ are the medium responses of Rayleigh and Love waves, respectively (Harkrider 1964), $u / w$ is the ellipticity of Rayleigh waves, $m$ and $n$ are the order of modes for Rayleigh and Love waves, $M$ and $N$ are the largest order of higher modes for Rayleigh and Love waves. Because the medium responses of higher modes, e.g., larger than the fifth mode, are much smaller than those of fundamental mode and only contribute in a short period range, without loss of generality, $M$ and $N$ are set to be 5 in the following calculations. Regarding the calculation of surface waves, the ellipticity, the medium response terms $A_{\mathrm{R}}$ of Rayleigh waves, and $A_{\mathrm{L}}$ of Love waves should be evaluated from the fundamental to the fifth higher mode, and for a given layered medium, Saito and Kabasawa (1993) proposed the "compound matrix" to overcome the numerical instability at high frequencies of the Thomson-Haskell propagation matrix. We improved their code and enabled it to evaluate Eq. (4). For a layered medium consisting of less than 10 layers, the computation of SW $H / V$ by our code on a desktop computer (64-bit, Windows 8.1, Intel Core i7, 3.20 GHz, RAM 8.0 GB) takes no more than $1 \mathrm{~s}$ even if the number of frequencies reaches 2000. It is significantly faster than the computation of FW $H / V$ by discrete wavenumber method (Sánchez-Sesma et al. 2011) which usually takes several or tens of minutes. It is also faster than the MATLAB runtime environment developed by GarcíaJerez et al. (2016) based on contour integration method which for the same problem takes several or tens of seconds. Furthermore, the numerical instability that can be observed at high frequencies of certain higher modes of Rayleigh waves for the García-Jerez et al. (2016)'s code is avoided.

\section{Approximation of FW $\mathbf{H} / \mathbf{V}$ for two-layered medium}

Harvey (1981) proposed that by adding a large velocity cap layer to a given layered medium and fixing the bottom, the Green's function of full-wave could be approximated with superposition of normal modes of surface waves. However, the effects of (1) depth of the upper interface, (2) the largeness of velocity of the cap layer, and (3) the impedance ratios of target layered medium, on the applicability of this approximation, were not discussed. In this section, we add a large velocity cap layer at large depth to examine the feasibility of approximation of the Green's function of full-wave with the Green's function of surface waves. This consideration is implicitly involved in the study of Arai and Tokimatsu (2004) who added two thick layers with large velocity to reach the engineering bedrock. They only formulated the $H / V$ of surface waves based on distributed surface sources assumption.

Table 1 lists the parameters of a two-layered medium consisting of a single layer and a half-space. We assume the $\mathrm{S}$-wave velocity $\left(V_{\mathrm{s}}\right)$ in the half-space is three times larger than that of the first layer, the density is fixed $\left(2.0 \mathrm{~g} / \mathrm{cm}^{3}\right)$ in each layer so that the impedance ratio is the same as velocity contrast, and P-wave velocity $\left(V_{\mathrm{p}}\right)$ is twice that of $V_{\mathrm{s}}$ in each layer, which implies a Poisson ratio of $1 / 3$. In any case, density and $\mathrm{P}$-wave velocity show 
Table 1 Target two-layered medium $\left(V_{p}=2 V_{s}\right.$, density $=2.0 \mathrm{~g} / \mathrm{cm}^{3}$ )

\begin{tabular}{lll}
\hline Layer & $\boldsymbol{V}_{\mathbf{s}}$ & Depth \\
\hline 1 & $V_{\mathrm{s} 1}=100 \mathrm{~m} / \mathrm{s}$ & $10 \mathrm{~m}\left(=\lambda_{0} / 4\right)$ \\
2 & $V_{\mathrm{s} 2}=3 V_{\mathrm{s} 1}$ & $\infty$ \\
\hline
\end{tabular}

much less sensitivity than $\mathrm{S}$-wave velocity to $H / V$ (e.g., Arai and Tokimatsu 2004; Lontsi et al. 2015). We regard this layered medium as "target" two-layered medium, and the layered medium composed of target layered medium and a cap layer as "cap-layered" medium.

First, we investigate the effect of depth of upper interface of cap layer on the applicability of approximating the FW $H / V$ of target two-layered medium with SW $H / V$ of cap-layered medium. Table 2 lists the parameters of caplayered media with three different depths (i.e., 2.5, 5, and 10 times the wavelength $\lambda_{0}$ at fundamental frequency or period) of upper interface of cap layers. For target twolayered medium, $\lambda_{0}$ is just four times the thickness of first layer, whatever the velocity and frequency is. The velocity of the cap layer is fixed, twice as large of the layer

Table 2 Cap-layered media with different depths of the upper interface of cap layer $\left(V_{p}=2 V_{s}\right.$, density $\left.=2.0 \mathrm{~g} / \mathrm{cm}^{3}\right)$

\begin{tabular}{|c|c|c|c|c|}
\hline Layer & $v_{s}$ & Depth & & \\
\hline 1 & $V_{\mathrm{s} 1}=100 \mathrm{~m} / \mathrm{s}$ & $10 \mathrm{~m}\left(=\lambda_{0} / 4\right)$ & & \\
\hline 2 & $V_{s 2}=3 V_{s 1}$ & $100 \mathrm{~m}\left(=2.5 \lambda_{0}\right)$ & $200 \mathrm{~m}\left(=5 \lambda_{0}\right)$ & $400 \mathrm{~m}\left(=10 \lambda_{0}\right)$ \\
\hline Cap & $2 V_{s 2}$ & $\infty$ & & \\
\hline
\end{tabular}

above the cap layer. As shown in the left panel of Fig. 1, the SW $H / V$ of the target two-layered medium is almost the same as FW $H / V$ in the period smaller than the predominant period of fundamental $\mathrm{S}$-wave resonance mode- $\mathrm{Tp}_{0}$, namely $0.4 \mathrm{~s}$, according to one quarter wavelength law. This indicates that the contribution of fullwave in the short period range is nearly the same as that of surface waves in the first layer. As shown in the middle panel of Fig. 1, the SW $H / V$ of cap-layered medium approximates well to the FW $H / V$ of target two-layered medium in the period ranging from $0.1 \mathrm{Tp}_{0}$ to $5 \mathrm{Tp}_{0}$ of the target medium if the depth of the upper cap layer is larger than $5 \lambda_{0}$. The reason is that the large velocity cap layer in the deep converts the body waves that are leaked from the first layer of target two-layered medium into higher modes of surface waves. It is obvious from Fig. 2 that the largest peak of SW $H / V$ of target two-layered medium is caused by a local trough in the vertical component which corresponds to the cutoff of vertical medium response of the first higher mode of Rayleigh waves. The cutoff of horizontal medium response has little effect on the largest peak, because its amplitude is not only smaller than that of fundamental mode of Rayleigh waves, but much smaller than that of Love waves. Due to the addition of cap layer, the vertical medium responses of the second, third and fourth higher modes of Rayleigh waves just fill in the local trough caused by the first higher mode, and this leads to a good approximation of FW $H / V$ of target two-layered medium with $\mathrm{SW} H / V$ of cap-layered medium. Therefore, although the main advantage of SW $H / V$ is its computation efficiency over FW $H / V$, the calculation of medium responses of surface waves gives

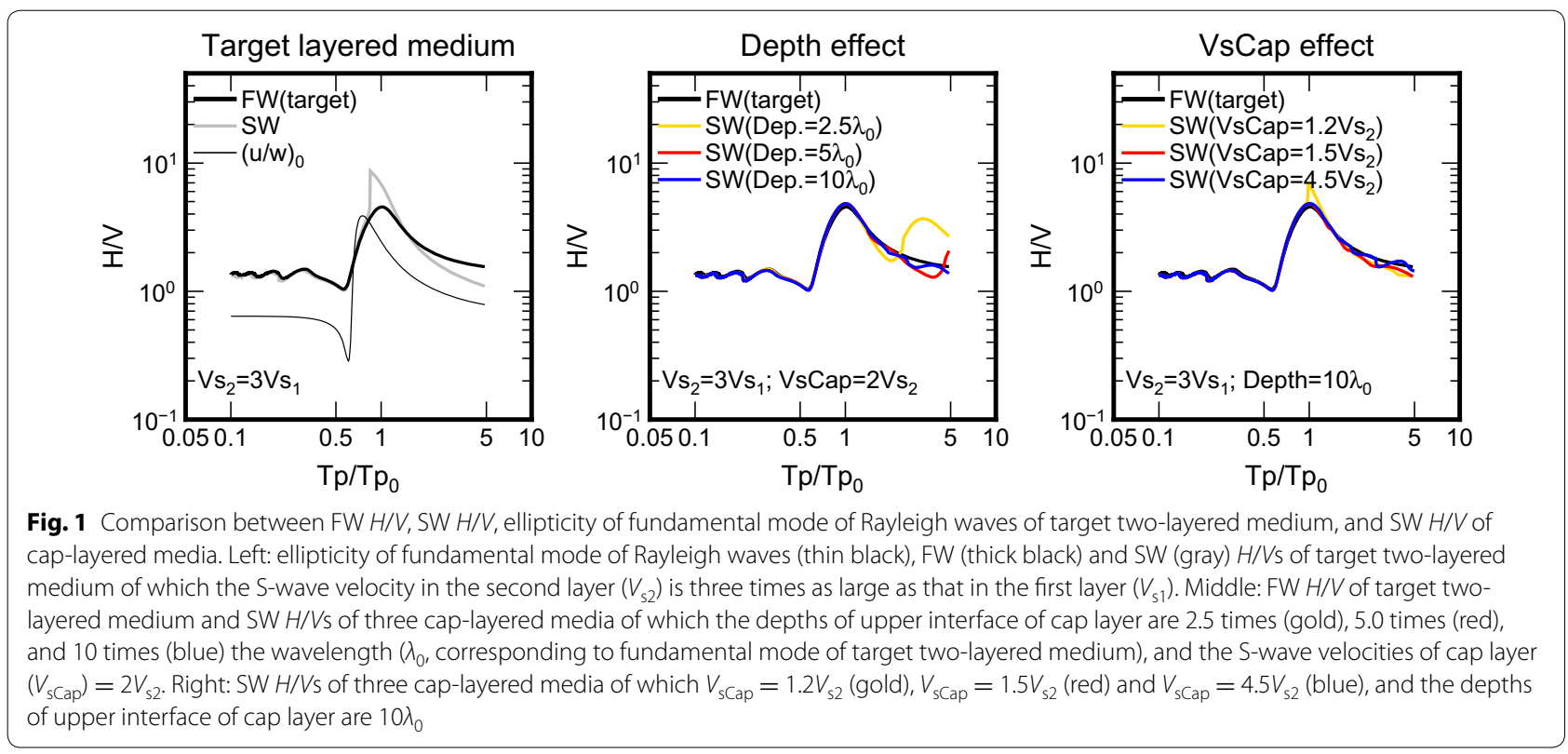



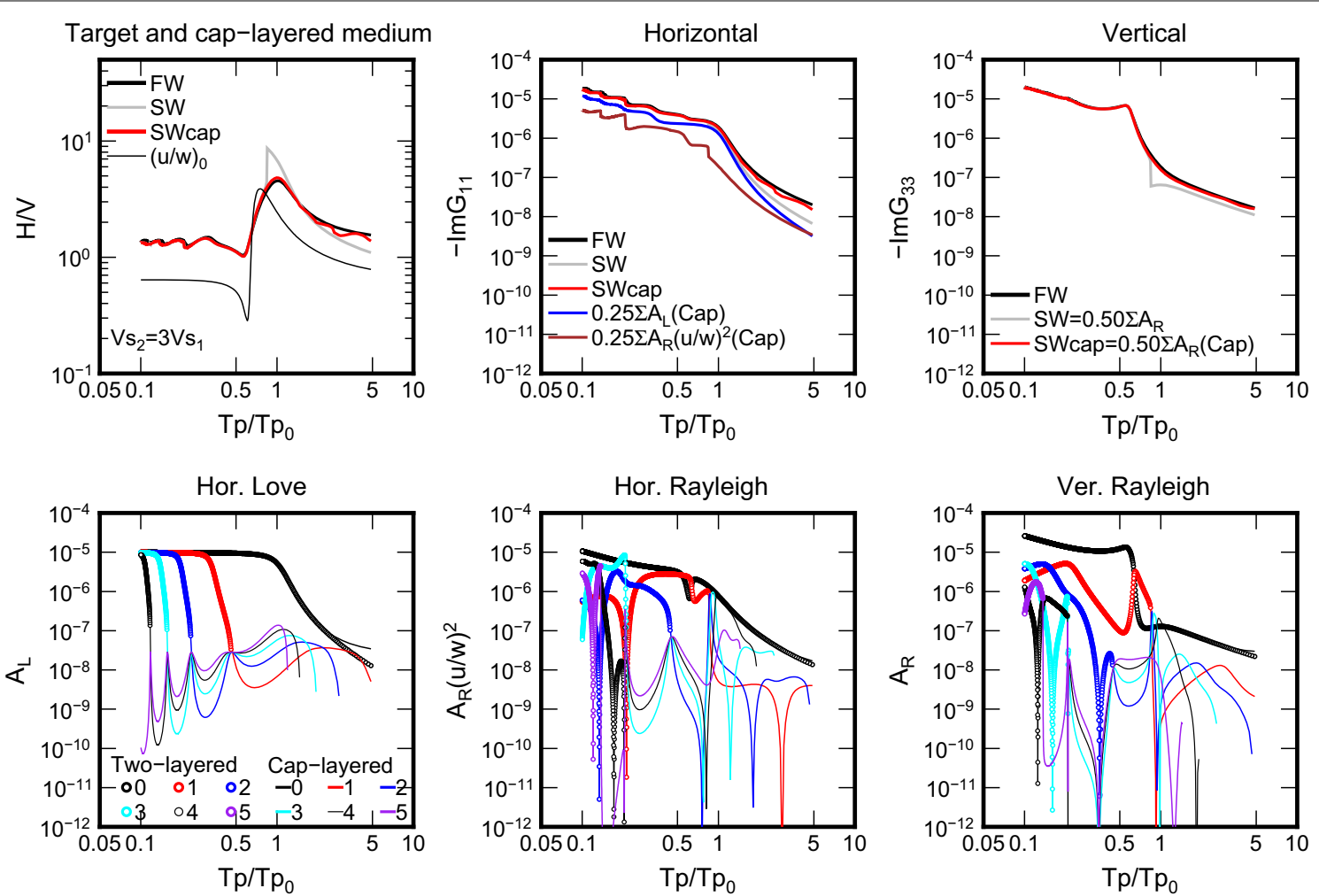

Fig. $2 \mathrm{H} / \mathrm{Vs}$, imaginary parts of the Green's functions, and medium responses of Love and Rayleigh waves of target two-layered $\left(V_{s 2}=3 V_{s 1}\right)$ and the corresponding cap-layered medium $\left(V_{\mathrm{sCap}}=2 V_{\mathrm{s} 2}\right.$, Depth $\left.=10 \lambda_{0}\right)$. Upper left: FW H/V (thick black), SW H/V (gray) and ellipticity (thin black) of fundamental mode of Rayleigh waves for target two-layered medium. Upper middle: imaginary parts of the Green's functions in the horizontal component for FW (thick black), SW (gray) of target two-layered medium, and for SW (red), Love waves (blue) and Rayleigh waves (brown) of cap-layered medium. Upper right: imaginary parts of the Green's functions in the vertical component for FW (thick black), SW (gray) of target twolayered medium, and for SW (red) of cap-layered medium. Lower left: medium responses (dot) of Love waves of target two-layered medium and those (solid curves) of cap-layered medium. Lower middle: horizontal medium responses (dot) of Rayleigh waves of target two-layered medium and those (solid curves) of cap-layered medium. Lower right: vertical medium responses (dot) of Rayleigh waves of target two-layered medium and those (solid curves) of cap-layered medium. The numbers denote the orders of normal modes of surface waves, e.g., 0 denotes the fundamental mode (black), 1 denotes the first higher mode (red), 2 the second higher mode (blue), 3 the third higher mode (cyan), 4 the fourth higher mode (thin black) and 5 the fifth higher mode (purple)

insight into the different contribution from fundamental and higher modes of both Rayleigh and Love waves.

Second, we investigate the effect of the cap layer velocity on the feasibility of approximating the FW $H / V$ of target two-layered medium with $\mathrm{SW} H / V$ of cap-layered medium. Table 3 lists the parameters of cap-layered media with different velocities of the cap-layer. The depth of upper interface of cap layer is set as $10 \lambda_{0}$ (i.e., $400 \mathrm{~m}$ here). As shown in the right panel of Fig. 1, the SW $H / V$ of cap-layered medium agrees well with the FW $H / V$ of target two-layered medium if the velocity of cap layer is larger than 1.5 times the velocity of the second layer $\left(V_{\mathrm{s} 2}\right)$. It is considered that the small velocity of cap layer (e.g., $1.2 V_{\mathrm{s} 2}$ ) is not enough for totally converting the body waves into surface waves.
Third, we investigate the effect of the impedance ratio of two-layered medium on the feasibility of approximating the FW $H / V$ of target two-layered medium with SW $H / V$ of cap-layered medium. Table 4 lists the parameters of target two-layered media with different impedance ratios. The depth of upper interface of cap layer is set to be $10 \lambda_{0}$, and the velocity of cap layer of these target twolayered media is twice as large of $V_{\mathrm{s} 2}$. Figure 3 shows that the amplitudes of FW and SW $H / V$ s increase with the impedance ratio. This fact prompted Nakamura to consider $H / V$ as a transfer function. Obviously, the impedance ratio is relevant. Ellipticity of the fundamental mode of Rayleigh waves is different from FW $H / V$, not only the amplitude but also the predominant period. For the Rayleigh's fundamental mode, the conspicuous period of 
Table 3 Cap-layered media with different velocities in the cap layer $\left(V_{p}=2 V_{s}\right.$, density $\left.=2.0 \mathrm{~g} / \mathrm{cm}^{3}\right)$

\begin{tabular}{|c|c|c|c|c|}
\hline Layer & $V_{s}$ & & & Depth \\
\hline 1 & $V_{\mathrm{s} 1}=100 \mathrm{~m} / \mathrm{s}$ & & & $10 \mathrm{~m}\left(=\lambda_{0} / 4\right)$ \\
\hline 2 & $V_{s 2}=3 V_{s 1}$ & & & $400 \mathrm{~m}\left(=10 \lambda_{0}\right)$ \\
\hline Cap & $1.2 V_{\mathrm{s} 2}$ & $1.5 \mathrm{~V}_{\mathrm{s} 2}$ & $4.5 V_{s 2}$ & $\infty$ \\
\hline
\end{tabular}

ellipticity coincides with the well-known local minimum in the vertical component. It is gradually close to that of FW $H / V$ as the impedance ratio increases. This fact was carefully studied in numerical simulations (Bonnefoy-Claudet et al. 2008). Similarly, for the SW $H / V$, the predominant periods of SW $H / V \mathrm{~s}$ of two-layered media for which the peaks coincide with the troughs of higher mode or fundamental mode (if the impedance ratio is large) of Rayleigh waves in the vertical component vary with impedance ratio. Figure 3 also shows that when the impedance ratio is not larger than 6 , the SW $H / V$ of caplayered medium agrees well with the FW $H / V$ of two-layered medium in the period range, e.g., $0.1 \mathrm{Tp}_{0}-5 \mathrm{Tp}_{0}$, even if only the contributions from fundamental to the fifth higher modes of surface waves are included in the SW $H / V$. Nolet et al. (1989) concluded that the evaluation of the Green's function requires 50-100 higher modes for locked-mode approximation. The site they selected is rather hard because the $V_{\mathrm{s}}$ in the first layer is as large as $1500 \mathrm{~m} / \mathrm{s}$. In contrast, the sites in our study are much softer, e.g., the $V_{\mathrm{s}}$ is about $300 \mathrm{~m} / \mathrm{s}$ in the first layer and is smaller than $1000 \mathrm{~m} / \mathrm{s}$ from the surface downward to $100 \mathrm{~m}$ in depth. This suggests that the Nolet et al. (1989)'s study was barely related with the results presented herein. The amplitudes of tiny fluctuations at very short periods, e.g., below $0.1 \mathrm{Tp}_{0}$, for $\mathrm{SW} H / V$ are slightly smaller than for FW $H / V$ due to the loss of modes higher than 5 for the target two-layered media. This small discrepancy between SW and FW $H / V$ s is difficult to detect for realistic multilayered media, such as those sites studied by Arai and Tokimatsu (2004) and Lunedei and Albarello (2009). Considering the temporal variation of microtremors, this small discrepancy seems negligible, e.g., smaller than one standard deviation of observed $H / V$, which we discuss later for one site. Besides, it only affects the accuracy of uppermost velocity structures (e.g., thinner than $1 \mathrm{~m}$ ) which is far from engineering interest. The amplitude of SW $H / V$ is twice as large as that of FW $H / V$ around the predominant period so that the difference cannot be neglected if the impedance ratio is larger than 6 .

Figure 4 shows the comparison between FW $H / V$ of target two-layered medium $\left(V_{\mathrm{s} 2}=8 V_{\mathrm{s} 1}\right)$ and $\mathrm{SW} H / V$ of cap-layered medium for which the depth of upper interface of cap layer is $10 \lambda_{0}$ (i.e., $400 \mathrm{~m}$ ), as well as the imaginary parts of the Green's functions and vertical medium responses from fundamental to the fifth higher modes. Due to cutoff effect of higher modes, the vertical medium responses from modes higher than third have no contributions at periods longer than the predominant period. Therefore, summation of higher modes larger than the fifth cannot improve the approximation of FW $H / V$ of target two-layered medium with SW $H / V$ of cap-layered medium at periods longer than the predominant period. We further place the cap layer at a great depth, such as $100 \lambda_{0}$ (i.e., $4000 \mathrm{~m}$ here) to investigate whether the SW $H / V$ of cap-layered medium can approximates well with FW $H / V$. Figure 5 shows the comparison between FW $H / V$ of target two-layered medium $\left(V_{\mathrm{s} 2}=8 V_{\mathrm{s} 1}\right)$ and $\mathrm{SW}$ $H / V$ of cap-layered medium for which the depth of upper interface of cap layer is as large as $100 \lambda_{0}$, as well as the imaginary parts of the Green's functions and vertical medium responses from fundamental to the 25th higher modes of Rayleigh waves. The discrepancy between FW $H / V$ of target medium and SW $H / V$ of cap-layered medium around the predominant period still cannot be neglected, as the vertical medium responses of higher modes of Rayleigh waves do not totally fill in the trough caused by the vertical medium response of fundamental mode. Therefore, even if the cap layer is located at a great depth and more modes are considered, the FW $H / V$ of target medium with large impedance ratio cannot be well approximated with SW $H / V$ of cap-layered medium. Tokimatsu and Tamura (1995) pointed out that if the impedance ratio is too large, the contribution of body waves is much larger than that of surface waves near the predominant period. It is thus considered that the cap layer fails to totally convert body waves into surface waves in this case. Applying rigid boundary condition

Table 4 Two-layered media with different impedance ratios and cap-layered media $\left(V_{p}=2 V_{s}\right.$, density $\left.=2.0 \mathrm{~g} / \mathrm{cm}^{3}\right)$

\begin{tabular}{|c|c|c|c|c|c|c|c|}
\hline Layer & $V_{s}$ & & & & & & Depth \\
\hline 1 & $V_{s 1}=$ & & & & & & $10 \mathrm{~m}\left(=\lambda_{0} / 4\right)$ \\
\hline 2 & $2 V_{s 1}$ & $4 V_{S 1}$ & $5 V_{s 1}$ & $6 V_{s 1}$ & $7 V_{s 1}$ & $8 V_{s 1}$ & $400 \mathrm{~m}\left(=10 \lambda_{0}\right)^{\mathrm{a}}$ \\
\hline Cap & $4 V_{s 1}$ & $8 V_{S 1}$ & $10 V_{s 1}$ & $12 V_{s 1}$ & $14 V_{s 1}$ & $16 V_{s 1}$ & $\infty$ \\
\hline
\end{tabular}

a The thickness of second layer is infinite for two-layered medium 

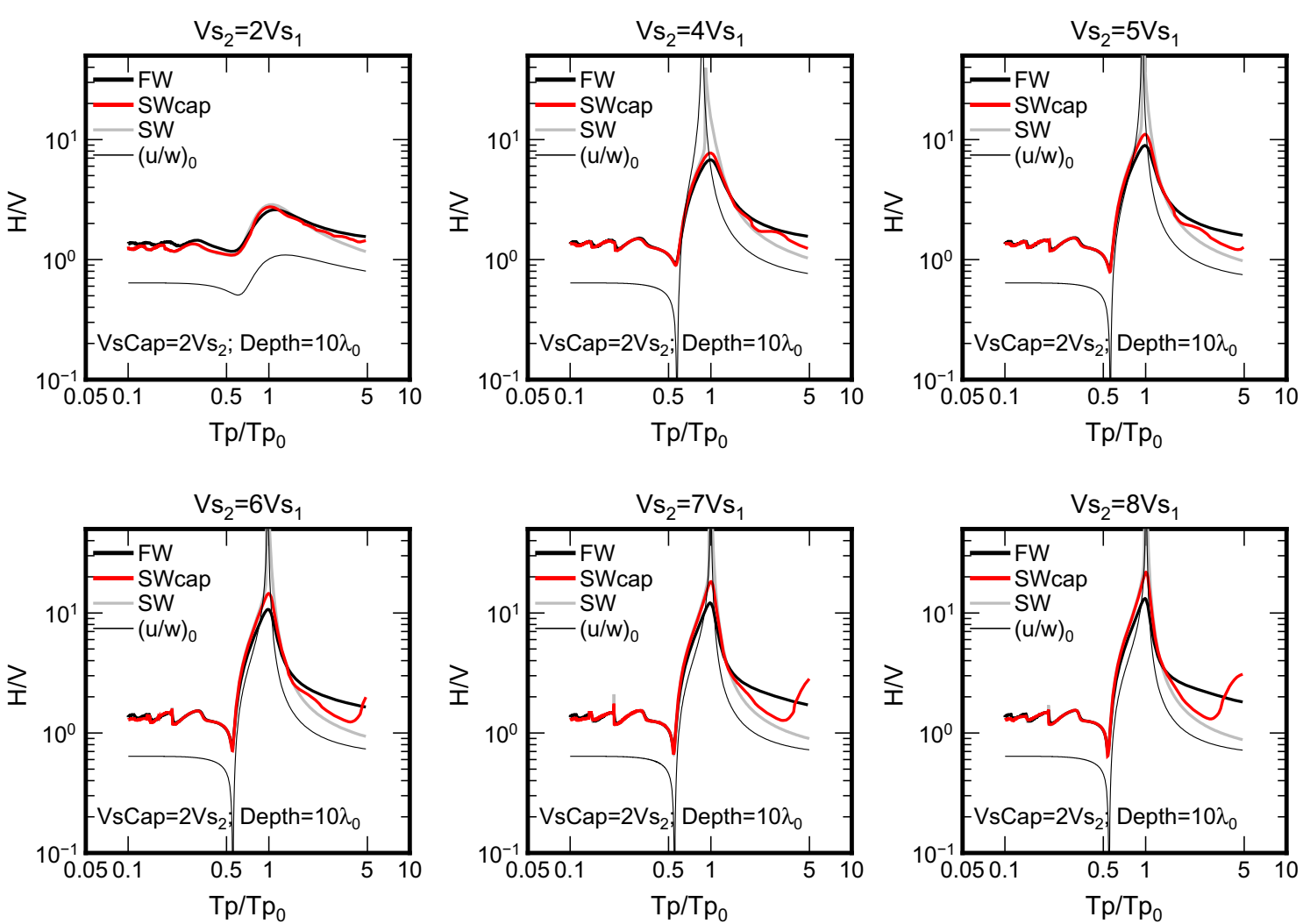

Fig. 3 Comparison between FW H/Vs (thick black), SW H/Vs (gray), ellipticity of fundamental mode of Rayleigh waves (thin black) of two-layered media with six different impedance ratios, and SW H/Vs (red) of corresponding cap-layered media (in all cases $V_{s c a p}=2 V_{s 2}$, Depth $=10 \lambda_{0}$ ). As for the two-layered media, $V_{s 2}=2 V_{s 1}$ in the upper left panel, $V_{s 2}=4 V_{s 1}$ in the upper middle panel, $V_{s 2}=5 V_{s 1}$ in the upper right panel, $V_{s 2}=6 V_{s 1}$ in the lower left panel, $V_{s 2}=7 V_{s 1}$ in the lower middle panel and $V_{s 2}=8 V_{s 1}$ in the lower right panel
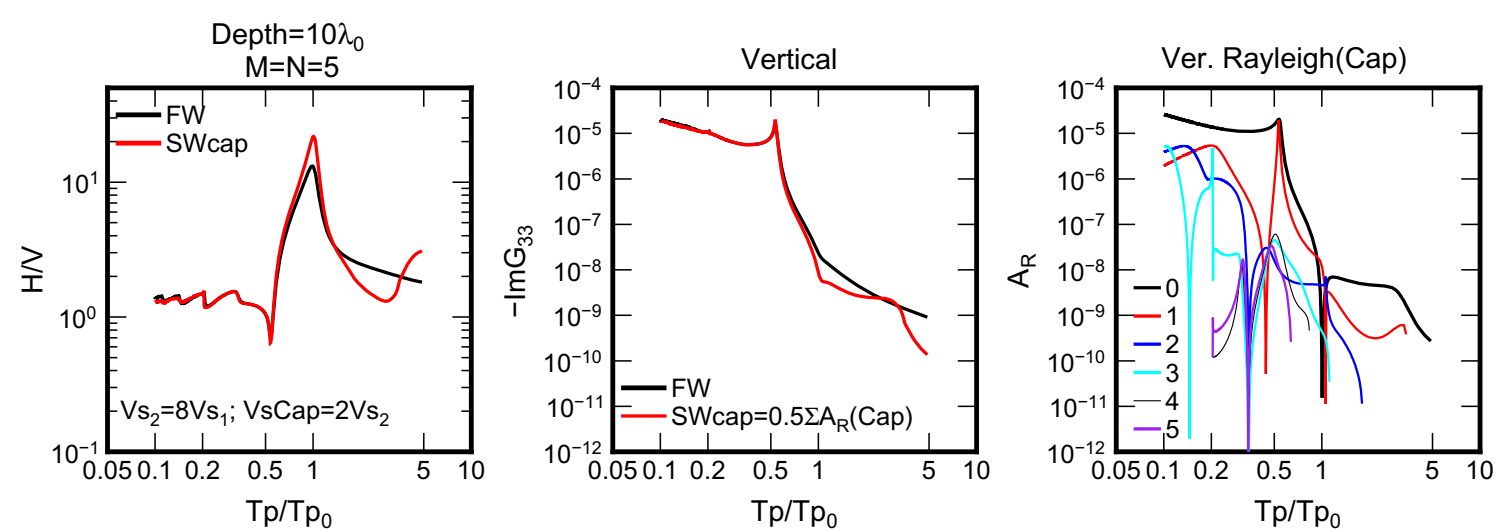

Fig. $4 \mathrm{H} / \mathrm{Vs}$, imaginary parts of the Green's function in the vertical component and vertical medium responses of Rayleigh waves from fundamental mode to fifth higher mode. Left: FW H/V (thick black) for target two-layered medium $\left(V_{s 2}=8 V_{s 1}\right)$, and SW H/V (red) for cap-layered medium $\left(V_{\text {sCap }}=2 V_{s 2}\right.$, Depth $\left.=10 \lambda_{0}\right)$. Middle: imaginary parts of the Green's function of FW (thick black) in the vertical component for target two-layered medium and that of SW (red) for cap-layered medium. Right: vertical medium responses of Rayleigh waves for fundamental mode (black), first higher mode (red), second higher mode (blue), third higher mode (cyan), fourth higher mode (thin black), and fifth higher mode (purple) 

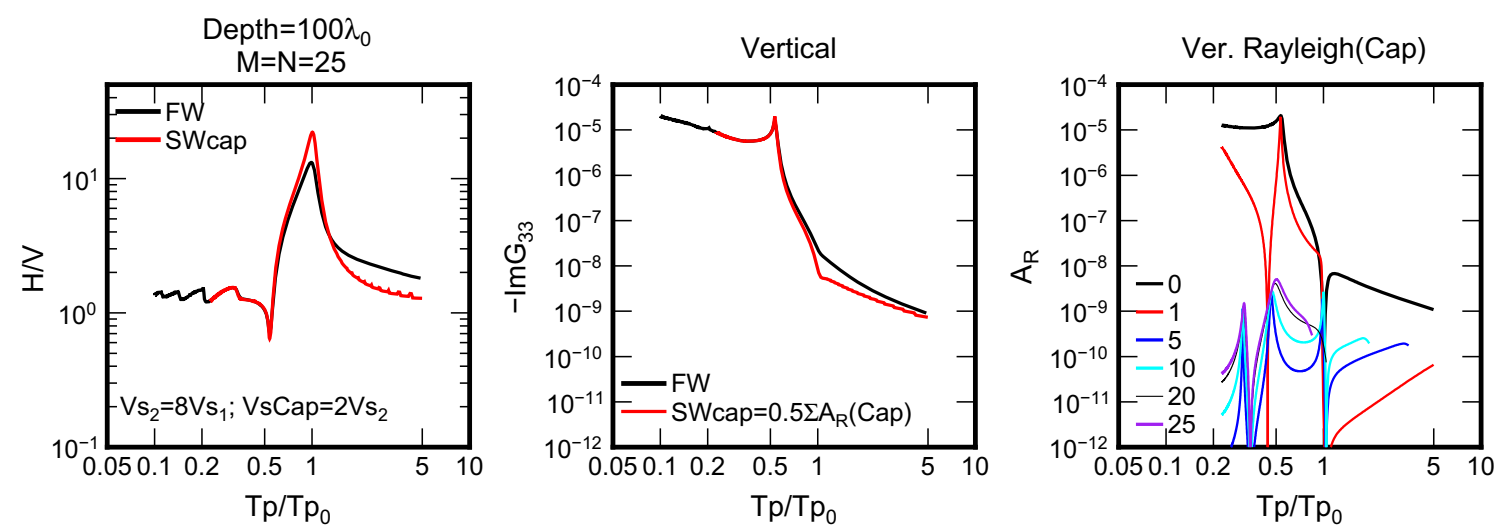

Fig. $5 \mathrm{H} / \mathrm{Vs}$, imaginary parts of the Green's function in the vertical component and vertical medium responses of Rayleigh waves from fundamental mode to 25 th higher mode. The depth of upper interface of cap layer is $100 \lambda_{0}$. Left: FW $H / V$ (thick black) for target two-layered medium $\left(V_{s 2}=8 V_{s 1}\right)$, and SW H/V (red) for cap-layered medium $\left(V_{\mathrm{sCap}}=2 V_{\mathrm{s} 2}\right.$, Depth $\left.=100 \lambda_{0}\right)$. Middle: imaginary parts of the Green's function of FW (thick black) in the vertical component for target two-layered medium and that of SW (red) for cap-layered medium. Right: vertical medium responses of Rayleigh waves for fundamental mode (black), first higher mode (red), fifth higher mode (blue), 10th higher mode (cyan), 20th higher mode (thin black), and 25th higher mode (purple). For clarity, medium responses are depicted for only six modes. The medium responses below $0.2 \mathrm{Tp}_{0}$ are omitted because the calculation becomes rather difficult when the dispersion curves of higher modes are quite close to one another at very short periods

(Margerin 2009) at the upper interface of cap layer is considered an effective solution to totally convert body waves into surface waves.

Another aspect that is of interest in practice is the effect of damping ratio or quality factor- $\mathrm{Q}$ which is used in the calculation of FW $H / V$ but not included in SW $H / V$. In the FW $H / V$, some damping was introduced by adding a small imaginary part to frequency and also using some material damping. Although the purpose was mainly to stabilize the results and to smooth the integrands in the wavenumber domain (Sánchez-Sesma et al. 2011), the practical interest of damping in realistic soil configurations led us to explore the effects of small material damping using a quality factor- $Q$. This is not accounted for in the theory, but in some cases adding small amount of damping allows for results closer to observations (e.g., Lawrence and Prieto 2011). We explore the effects of quality factor- $Q$ on FW $H / V$ s of two-layered media with three different impedance ratios shown in Fig. 6. It suggests that the difference of FW $H / V$ s caused by three different $Q$ values (50, 100 and 500) is negligible. Thus, the discrepancy between FW $H / V$ of target medium with large impedance ratio and SW $H / V$ cannot be attributed to the damping ratio or $Q$ values.

Finally, it should be noted that the large velocity cap layer at large depth is just an artificial layer used to convert the body waves leaked from the bottom of given layered medium into surface waves of cap-layered medium. Meanwhile, the SW $H / V$ of cap-layered medium is regarded a simplified calculation of FW $H / V$ of layered
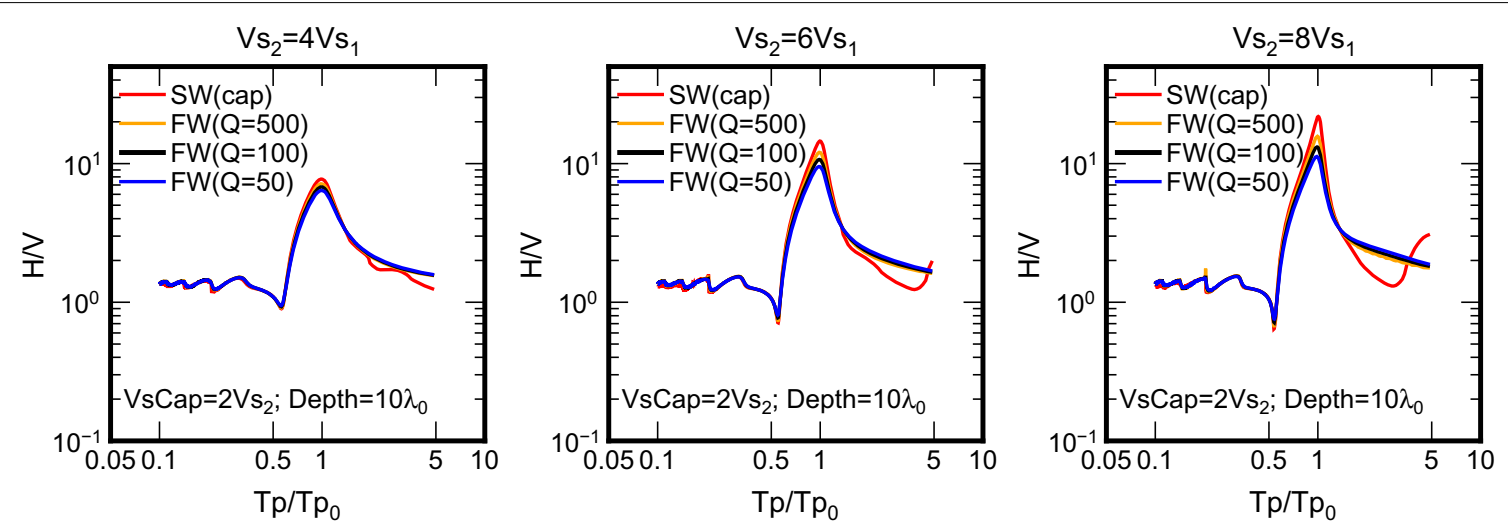

Fig. 6 FW H/Vs of target two-layered media with different impedance ratios (Left: $V_{s 2}=4 V_{s 1}$, Middle: $V_{s 2}=6 V_{s 1}$, Right: $V_{s 2}=8 V_{s 1}$ ) for three different $Q$ values $\left(Q=500\right.$, orange; $Q=100$, black; $Q=50$, blue), and $S W($ red $) H / V$ s of cap-layered media $\left(V_{s c a p}=2 V_{s 2}\right.$, Depth $\left.=10 \lambda_{0}\right)$ 
medium, and we agree that microtremor consists of fullwave, but not surface waves only. Based on the aforementioned discussions, we conclude that the FW $H / V$ of target layered medium should be well approximated with SW $H / V$ of cap-layered medium in the period range, e.g., $0.1 \mathrm{Tp}_{0}-5 \mathrm{Tp}_{0}$, under the following conditions: The impedance ratio of target layered medium is not larger than 6, and the $S$-wave velocity of cap layer is about twice as large as that in the half-space of target layered medium, the depth of upper interface of cap layer is about $(5-10) \lambda_{0}$. However, it should be noted that $Q=100$ might be too large for FW $H / V$ at soft sediment sites, and thus further investigation is needed.

\section{Approximation of $\mathrm{FW} \mathbf{H} / \mathbf{V}$ for multilayered medium}

In Japan, PS logging data between surface and bottom of boreholes at most KiK-net strong-motion stations are available, so the underground velocity structures of PS logging data are adopted to generate multilayered media. With respect to the corresponding cap-layered media, we set the velocity of the cap layer as about two times larger than that in the above layer. Because one cannot precisely estimate the $\mathrm{Tp}_{0}$ or $\lambda_{0}$ for a given layered medium, we define the "apparent" predominant period of S-wave fundamental mode $\left(\mathrm{Tp}_{0}^{\mathrm{A}}\right)$ as the ratio of the "apparent" wavelength of fundamental mode $\left(\lambda_{0}^{\mathrm{A}}\right)$ for the given layered medium to the thickness-weighted average $S$-wave velocity (Fäh et al. 2001). The "apparent" wavelength of fundamental mode $\left(\lambda_{0}^{\mathrm{A}}\right)$ is four times the depth from surface to the uppermost interface of half-space, but not four times the thickness of first layer anymore. Both $\mathrm{Tp}_{0}^{\mathrm{A}}$ and $\lambda_{0}^{\mathrm{A}}$ become the "real" $\mathrm{Tp}_{0}$ and $\lambda_{0}$ for the two-layered medium (single layer over a half-space) and tend to be the "real" ones if the velocity contrast at the interface of half-space is large enough for multilayered medium. We find that if the depth of cap layer is set to be about (5-10) $\lambda_{0}^{\mathrm{A}}$, the $\mathrm{SW} H / V$ of cap-layered medium approximates well to the FW $H / V$ of target multilayered medium. Six KiK-net stations were selected after a careful scrutiny that accounts for local geology, seismicity, and site effects. They do not locate near the edge between basin and mountain nor close to active faults so that the 3D effects are considered minor. Figure 7 shows the $\mathrm{S}$-wave velocity profiles in the top panels at six KiK-net stations. Table 5 lists the basic parameters, such as $\mathrm{Tp}_{0}^{\mathrm{A}}$, thicknessaveraged $V_{s}$, and $\lambda_{0}^{\mathrm{A}}$ for velocity profiles at these stations. The numerical values show the $\mathrm{Tp}_{0}^{\mathrm{A}}$ at the interface of half-space of target layered medium in black and that at the uppermost interface of cap layer in red. It is obvious that the cap layer at large depth produces a $\mathrm{Tp}_{0}^{\mathrm{A}}$ about 5-10 times as larger as that for target layered medium. These 1D vertical profiles are obtained from PS logging data. In most circumstances, that 1D profile is verified by studying the measured amplification from an earthquake, such as comparing the observed surface/bottom of borehole spectral ratio with theoretical 1D transfer function (e.g., Thompson et al. 2012). Another approach is to use microtremor to accomplish such verification by comparing the observed microtremor $H / V$ with the theoretical $H / V$, considering that the soil models are 1D or only depth dependent at these stations. The underground velocity structures consisting of PS logging data are used to evaluate four theoretical $H / V \mathrm{~s}$ in the bottom panels, i.e., ellipticity of fundamental mode of Rayleigh waves, FW $H / V$ and SW $H / V$ of the multilayered medium, and $\mathrm{SW} H / V$ of the corresponding cap-layered medium. The coincidence between FW and SW $H / V$ s of multilayered media in the short period range at all stations again indicates that the contribution of surface waves which are trapped in the shallow layers is overwhelmingly dominant. By just adding a cap layer to the bottom of borehole, the SW $H / V$ s of cap-layered media approximate the FW $H / V$ s well at almost all the stations except KMMH14 at which a relatively large impedance ratio near the bottom of borehole could be responsible for the discrepancy between FW $H / V$ of multilayered medium and SW $H / V$ of cap-layered medium. However, this discrepancy is negligible given that the microtremor is subjected to the temporal variance (Okada 2003).

The ellipticities of fundamental mode of Rayleigh waves at all stations have two peaks which are caused by the underground velocity structures of shallow layers, and deep layers, respectively. The sharp peak at periods shorter than $0.5 \mathrm{~s}$ at TCGH16 station corresponds to the large impedance ratio in the shallow layers as shown in the S-wave velocity profiles. The sharp peaks at periods

(See figure on next page.)

Fig. 7 Profiles of initial underground velocity structures (upper) and H/VS (lower) at six KiK-net strong-motion stations, i.e., TCGH16, KMMH03, GIFH28, GIFH29, SZOH26, and KMMH14. In the upper panels, solid line shows the profile of PS logging data, dot line shows the half-space of PS logging data, and dashed line shows the cap layer. The numerical values show the "apparent"fundamental predominant period ( $\left.\operatorname{Tp}_{0}^{A}\right)$ at the interface of half-space of target layered medium in black and that at the uppermost interface of cap layer in red. In the lower panels, thick black curves denote the observed $H / V$ s, dashed curves show the observed $H / V$ within one standard deviation, thin black curves denote the ellipticity of fundamental mode of Rayleigh waves, cyan curves denote the FW H/Vs, blue curves denote the SW H/Vs of layered media consisting of PS logging data, and red curves denote the SW H/VS of cap-layered media 

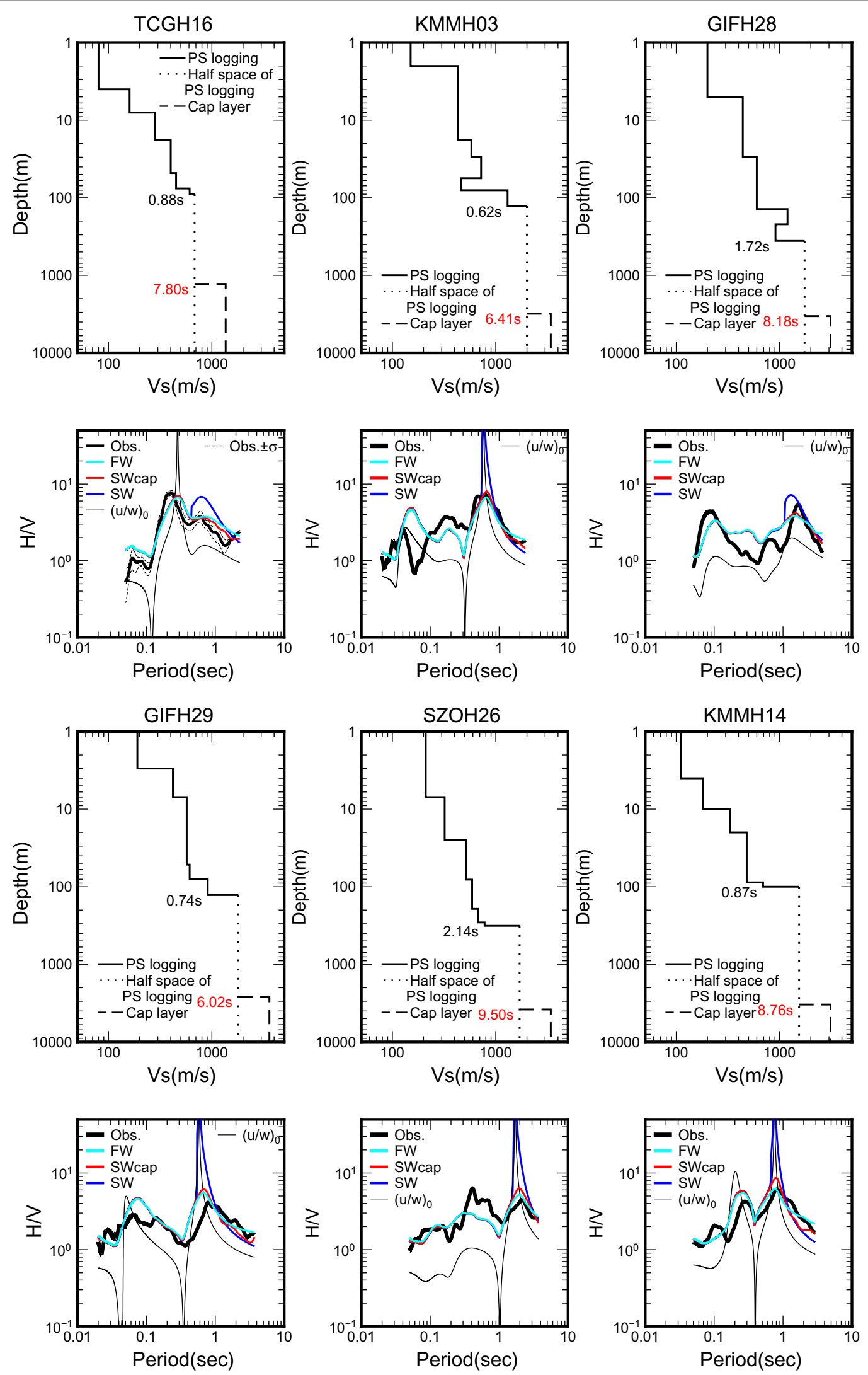
longer than $0.5 \mathrm{~s}$ at other stations correspond to the large impedance ratio in the deep layers near the bottom of boreholes. Both peaks of ellipticity of fundamental mode of Rayleigh waves at GIFH28 are moderate. This is attributed to the moderate impedance ratio from surface to bottom of borehole.

The observed microtremor $H / V$ s at six KiK-net stations are also shown in bottom panels of Fig. 7. We conduct the microtremor measurement with a three-component velocity seismometer, a GPS receiver, and a recorder with 24 bits. We conduct the measurement for 30-60 min with $100 \mathrm{~Hz}$ sampling at all stations except TCGH16 where we observed as long as $12 \mathrm{~h}$. We remove those time windows $(\mathrm{Tw}=40.96 \mathrm{~s})$ including extremely large amplitude which is caused by nearby human activities, such as jogging, vehicles, before obtaining the power spectra in three components. These power spectra in three components are then averaged over the number of time windows. The observed $H / V$ is obtained from the square ratio of average power spectra in the horizontal components (arithmetic summation of north-south (NS) and east-west (EW) components) to that in the vertical component, just as proposed by Arai and Tokimatsu (2004) and numerically verified by Albarello and Lunedei (2013). Smoothing with $b=50$ by Konno and Ohmachi (1998) is finally implemented on the observed $H / V$. Thus only one $H / V$ without temporal variability can be obtained at one site. As the record is long at TCGH16, we divide it into 24 parts each of which is used to evaluate $H / V$ to investigate the temporal variation of observed $H / V$. We take the average $H / V$ as the observed one at TCGH16 and find the standard deviation at each period. The average $H / V$ is shown in thick black curve, and the ones obtained from the average plus and minus one standard deviation are shown in dashed curves in Fig. 7. Temporal variation can be observed, yet it is very small. The mismatch between the FW $H / V$ and observed $H / V$ suggests that the underground velocity structures of PS logging data are not fully reliable and thus should be improved through inversion of $H / V$.

\section{Identification of underground velocity structures from SW HIV}

Since the computation of medium responses of surface waves is significantly faster than the computation of full-wave, and FW $H / V$ can be approximated with SW $H / V$ by just adding a cap layer to the bottom of given layered medium, we prefer to identify the underground velocity structures from $\mathrm{SW} H / V$. We perform the identification by simulated annealing algorithm which is elaborated in Satoh (2006). Generally, this algorithm finds many local minima at the beginning and gradually converges to a global minimum. The temperature scheme is expressed as $T_{k}=T_{0} \cdot \exp \left(-c k^{\alpha}\right)$, where $k$ is the step of cooling, $T_{k}$ is the temperature at the $k$ th step, $T_{0}$ is the initial temperature, and $c$ and $\alpha$ are key parameters controlling the cooling speed. We set $T_{0}, c$, and $\alpha$ to be 1.0, 1.0, and 0.6, respectively, iterations at one step to be 5 , and total steps 1000 . The objective function $E_{m}$ is defined as follows: (Saguchi et al. 2009)

$$
E_{m}=\frac{\sum_{f_{\text {min }}}^{f_{\text {max }}}\left[\left|(H / V)_{f}^{\mathrm{SW}}-(H / V)_{f}^{\mathrm{obs}}\right| / f\right]}{\sqrt{\sum_{f_{\min }}^{f_{\max }}\left[(H / V)_{f}^{\mathrm{SW}} / f\right]} \cdot \sqrt{\sum_{f_{\min }}^{f_{\text {max }}}\left[(H / V)_{f}^{\mathrm{obs}} / f\right]}},
$$

where $(H / V)_{f}^{\mathrm{SW}}$ denotes the SW $H / V$ of cap-layered medium, $(H / V)_{f}^{\text {obs }}$ denotes the observed $H / V, f$ denotes frequency (equally spaced precisely as it is in FFT), $f_{\min }$ and $f_{\max }$ denote the minimum and maximum frequencies of the target fitting frequency range, respectively. The $f_{\min }$ is determined in consideration of the depth of underground velocity structures from surface to bottom, e.g, it may be not deep enough to explain peak at low frequency or long period. The $f_{\max }$ is the Nyquist frequency- $50 \mathrm{~Hz}$, if sampling frequency is $100 \mathrm{~Hz}$, whereas $f_{\max }=20 \mathrm{~Hz}$ is better for some sites. In this study, we set $f_{\min }$ to be $0.25-0.5 \mathrm{~Hz}$, and $f_{\max }$ to be $20-50 \mathrm{~Hz}$. The weight function is usually employed in the objective function to guarantee a good fitting over a broad frequency range. Typically, the expressions for the objective or cost functions are weighted sums of residuals. The choice of the weights is a matter of taste. Certainly, a common selection aimed to smooth out the results is to use frequency so that larger frequencies with their multiple oscillations have less constraining power. Our previous studies (Wu et al. 2012, 2016; Wang et al. 2017) suggested that the linear "frequency" worked well as the weight function, while Satoh (2006) suggested a logarithmic frequency to be another weight function. The temporal variation of observed $H / V$ is considered small, and it is not included in the objective function. The discussion about the effect of different weight functions on the objective function and how to incorporate the temporal variation in the objective function is important, yet beyond the scope of this study.

If there is not any a priori knowledge about the underground velocity structures, the identified velocity structures are not unique by inversion of $H / V$ only. It is a good practice to implement the joint inversion of both $H / V$ and dispersive curve, such as Parolai et al. (2005), Picozzi et al. (2005), Arai and Tokimatsu (2005), and Piña-Flores et al. (2017). An alternative method to uniquely identify the underground velocity structures by the inversion of $H / V$ only is to make use of a priori knowledge. In this study, we refer to the PS logging data to be the initial underground velocity structures rather than those generated by genetic algorithm (Piña-Flores et al. 2017). It 
Table 5 Basic parameters of velocity profiles at six KiK-net stations

\begin{tabular}{lcccccr}
\hline & TCGH16 & KMMH03 & GIFH28 & GIFH29 & SZOH26 & KMMH14 \\
\hline$\lambda_{0}^{A}(\mathrm{~m})$ & 360.0 & 512.0 & 1440.0 & 512.0 & 1276.0 & 400.0 \\
Ave- $V_{s}(\mathrm{~m} / \mathrm{s})^{\mathrm{a}}$ & 407.4 & 830.5 & 835.0 & 692.7 & 596.1 & 457.4 \\
$T p_{0}^{\mathrm{A}}(\mathrm{s})$ & 0.88 & 0.62 & 1.72 & 0.74 & 2.14 & 0.87 \\
\hline
\end{tabular}

a Thickness-averaged S-wave velocity

is well known that the accuracy of velocity structures of PS logging data in shallow layers is generally not reliable enough. The velocities of soil layers near the surface in most cases are unstable and fluctuate, and the interface between two adjacent layers is not easy to detect and so is the layer thickness. As shown in Fig. 7, the $H / V$ s for initial cap-layered media match well with the observed ones at relative long periods, such as around $0.6 \mathrm{~s}$ at TCGH16, $0.6 \mathrm{~s}$ at KMMH03, $2.0 \mathrm{~s}$ at SZOH26, and $0.8 \mathrm{~s}$ at KMMH14, respectively. This is attributed to the fact that thickness and average velocity from surface downward to the layer boundary which has a remarkable velocity contrast at each site is clearly detectable for PS logging data. It implies that the velocity structures in moderate depth accounting for the $H / V \mathrm{~s}$ at relative long periods are reliable. Thus we fix the average velocity down to the moderate depth (including the bottom of the boreholes) during the identification. Moreover, considering the density and $V_{\mathrm{p}}$ are insensitive to $H / V$, we fix the densities and the Poisson ratios so that $V_{\mathrm{p}}$ can be evaluated from identified $V_{\mathrm{s}}$. Under these constraints, we are able to uniquely identify the velocity structures in the shallow layers from our SW $H / V$ for cap-layered media. Although there is a trade-off relationship between $V_{\mathrm{s}}$ and thickness, identification of both $V_{\mathrm{s}}$ and thickness in each layer converges faster than that of only $V_{\mathrm{s}}$ or only thickness in each layer at some stations.

Figure 8 shows all the profiles of identified $V_{\mathrm{s}}$ structures between the surface and bottom of boreholes as well as the initial ones, and the observed and theoretical $H / V \mathrm{~s}$. The initial underground velocity structures are composed of those PS logging data and a cap layer. Additional file 1: Tables A1-A6 list the initial and identified underground velocity structures at six KiK-net stations, while the PS logging data used to construct the initial underground velocity structures are available online (see "Availability of data and materials"). The large variance of velocity profiles and corresponding SW $H / V \mathrm{~s}$ (shown in light pink) is explained that the simulated annealing algorithm is likely to find many solutions at local minima before the solution converges toward a global minimum. The black numerical values in the left bottom of $H / V$ panels are the objective function Em between observed $H / V \mathrm{~s}$ and SW $H / V \mathrm{~s}$ for initial underground velocity structures. The red numerical values in the left bottom of $H / V$ panels are the objective function Em between observed $H / V \mathrm{~s}$ and $\mathrm{SW}$ $H / V$ s for identified underground velocity structures. The smaller Em values suggest that the SW $H / V$ s for identified underground velocity structures agree better with the observed $H / V \mathrm{~s}$ at these stations than SW $H / V \mathrm{~s}$ for initial underground velocity structures. Meanwhile, the FW $H / V$ s, evaluated with identified underground velocity structures without cap layer, are in good agreement with SW $H / V \mathbf{s}$ of corresponding cap-layered media. It in turn suggests that the SW $H / V$ of cap-layered medium can be considered as a simplified calculation of FW $H / V$ of layered medium without cap layer, to identify the underground velocity structures.

\section{Comparison with previous study on $H / V$ and validation of identified underground velocity structures}

Regarding SW $H / V$, we studied the differences between the diffuse field approximation (DFA-SW $H / V$ ) and the Arai and Tokimatsu's (2004) proposal that considers surface waves from a random distribution of surface

\footnotetext{
(See figure on next a page.)

Fig. 8 Profiles of underground velocity structures between the surface and the bottom of boreholes (upper) and H/Vs (lower) at six KiK-net strongmotion stations. In the upper panels, thin black lines show the profiles of initial underground velocity structures, light pink lines show the profiles of underground velocity structures identified during all the iterations, and thick black lines show the profiles of identified underground velocity structures. In the lower panels, thick black curves denote the observed H/Vs, thin black curves denote the SW H/Vs of initial cap-layered media, light pink curves denote the SW H/Vs of all identified cap-layered media during the iterations, red curves denote the SW H/Vs of identified cap-layered media, and cyan curves denote the FW H/Vs of identified layered media. The numerical values in black represent the objective function Em between observed H/VS and SW H/Vs of initial cap-layered media, and the numerical values in red represent the objective function Em between observed H/Vs and SW H/Vs of identified cap-layered media
} 

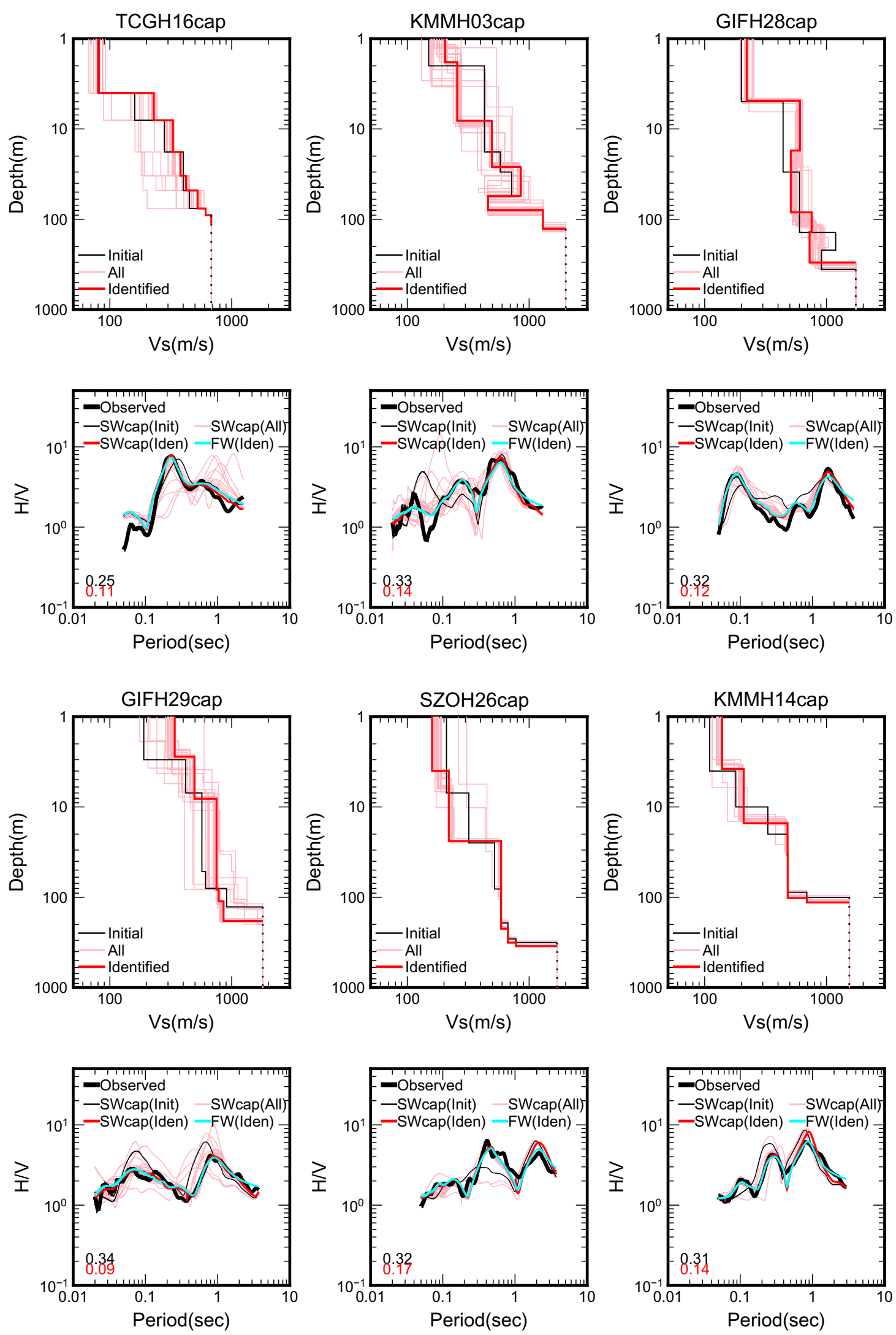


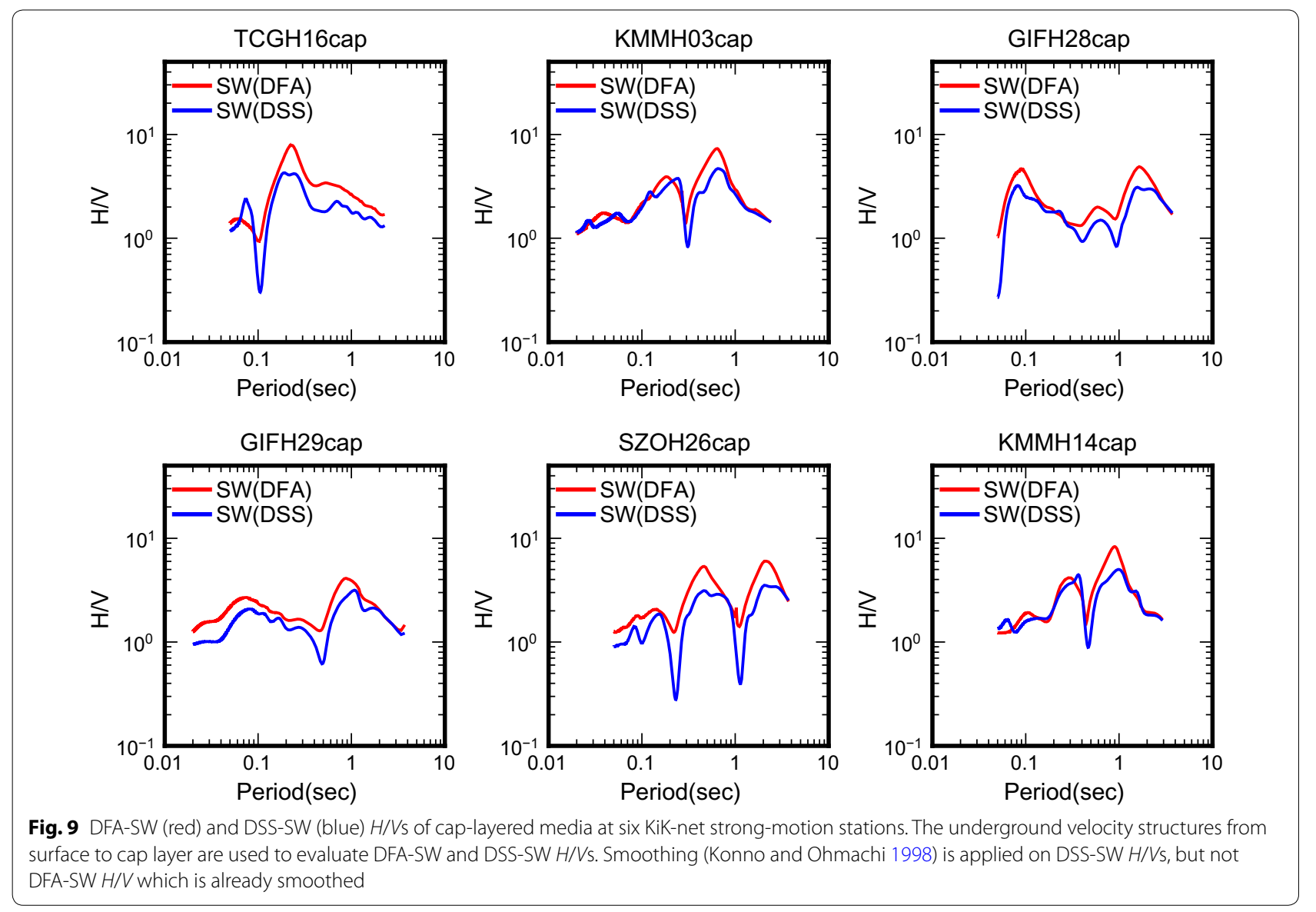

sources (DSS-SW $H / V$ ). Figure 9 shows the comparison between (DFA) SW and DSS-SW $H / V$ s. Just as we did on the observed $H / V \mathrm{~s}$, smoothing with $b=50$ by Konno and Ohmachi (1998) is implemented on the DSS-SW $H / V \mathbf{s}$, but not on (DFA) SW $H / V$ s as they are already smoothed. Both of these two SW $H / V$ s at six stations show a qualitative agreement. It is attributed to the fact that both of these two studies incorporate the contributions of both fundamental and higher modes of surface waves (e.g., the medium response terms of $A_{\mathrm{R}}$ and $A_{\mathrm{L}}$ ), despite of different premises. On the other hand, the amplitudes of DSSSW $H / V$ s at peaks are a bit smaller than those of SW $H / V$ s. It agrees with the study of Kawase et al. (2015) who compared the DSS-SW and FW $H / V$ s by use of underground velocity structures identified from DSS-SW $H / V$ s. The constant Rayleigh-to-Love waves amplitude ratio for horizontal motions $(\mathrm{R} / \mathrm{L})$ in a period range of $0.1-5 \mathrm{~s}$ which is assumed to by Arai and Tokimatsu (2004) suggests that the shape of DSS-SW $H / V$ is the same as $H / V$ evaluated from the medium responses of fundamental and higher modes of Rayleigh waves, and the different amplitude between them only relies on the value of $\mathrm{R} / \mathrm{L}$. In fact, the $\mathrm{R} / \mathrm{L}$ may vary with site condition, as reviewed by Bonnefoy-Claudet et al. (2006b). The apparent peaks of DSS-SW $H / V$ around $0.07 \mathrm{~s}$ at TCGH16 and $0.4 \mathrm{~s}$ at KMMH14, and sharp troughs imply the limitation of $\mathrm{R} / \mathrm{L}$ which should be variable with period rather than constant. Arai and Tokimatsu (2004) assumed that the source-to-receiver distance is larger than one wavelength. Thus their DSS-SW $H / V$ involves the power spectra of displacements in the horizontal components $\left(G_{13}\right.$ and $G_{23}$ ) excited by vertical loading force, and the power spectra of displacement in the vertical component $\left(G_{31}\right.$ and $G_{32}$ ) excited by horizontal loading force. In contrast, the imaginary parts of $G_{13}, G_{23}, G_{31}$, and $G_{32}$ which are not required for evaluating FW $H / V$ in Eq. (1) are zero when source and receiver coincide with each other both in position and direction. It is considered another reason for the difference between (DFA) SW and DSS-SW H/Vs. Lunedei and Albarello (2009) followed the assumption of Arai and Tokimatsu (2004) that surface waves were generated by a random distribution of surface sources, and improved the formulation for surface waves approximation by considering the effects of damping and discarding the $\mathrm{R} / \mathrm{L}$ assumption. They further released the limitation on the source-to-receiver distance (e.g., $r=0$ ) to develop 
the formulation for full-wave (Lunedei and Albarello 2010). For the same layered medium, DSS-FW $H / V$ is found to be similar to FW $H / V$ (García-Jerez et al. 2012; Lunedei and Albarello 2015), but the computation is not faster than that of FW $H / V$ evaluated by discrete wavenumber method.

The available strong-motion records at KiK-net stations can be used to evaluate the Earthquake motions $H / V$ Spectral Ratio (EHVSR) and Spectral Ratio of Horizontal motions between Surface and Bottom of borehole (SRHSB). Therefore, we validate the underground velocity structures identified from SW $H / V$, if the observed and theoretical EHVSRs and SRHSBs have a good consistency. Data analysis about earthquake motions is similar to that of Ducellier et al. (2013). The S-wave parts of strong-motion records for small events (e.g., peak ground acceleration < 100 gal; magnitude $<6$; epicenter distance $<200 \mathrm{~km}$ ) are used to evaluate the power spectra in three components, i.e., north-south, east-west, and up-down. Smoothing with $b=50$ by Konno and Ohmachi (1998) is implemented on the power spectra in three components before finding the square root of power spectral ratios, EHVSR and SRHSB, for each small event. Finally, the observed EHVSRs and SRHSBs are arithmetically averaged over the number of small events. The average EHVSRs and SRHSBs, as well as one standard deviation plus and minus the average one, are shown in Fig. 10. Additional file 2: Tables B1-B6 list the information about small events at six KiK-net stations. Although the number of strong-motion records at a couple of stations is not adequate due to low seismicity, we provide the standard deviations of spectral ratios as rough indicators of variability. The underground velocity structures from surface to bottom of boreholes are used to evaluate the theoretical SRHSBs by the propagation matrix method (Haskell 1960). The underground velocity structures from surface to half-space without cap layer are used to evaluate the theoretical EHVSR which is proportional to the square ratio of transfer functions between S- and P-waves based on diffuse field approximation (Kawase et al. 2011). Smoothing with $b=50$ by Konno and Ohmachi (1998) is also implemented on the theoretical SRHSBs and transfer functions.

The effects of attenuation are present in real data, but using damping within the diffuse field approximation is not fully supported by theory. Snieder et al. (2007) showed that the Green's function can be retrieved from the response to random forcing for a variety of conditions, including the extreme case of the diffusion equation. In many practical circumstances, small amounts of damping allow stabilizing results so that the retrieved Green's functions are consistent with observations (e.g., Lawrence and Prieto 2011). Here we assume the quality factor for S-wave, $Q s=100$, the same as that for FW $H / V$. Figure 10 depicts the comparison between observed and theoretical EHVSRs and SRHSBs. The theoretical counterparts were computed for both initial and identified underground velocity structures. The black numerical values in the left bottom of EHVSR and SRHSB panels are the objective functions between observed and theoretical spectral ratios for initial underground velocity structures, and the red ones are the objective function between observed and theoretical spectral ratios for identified underground velocity structures. The smaller objective functions at stations other than GIFH29 and KMMH14 suggest that EHVSRs and SRHSBs for identified underground velocity structures agree better with the observed counterparts. This indicates that the underground velocity structures identified from SW $H / V$ are reasonable. The disagreement between observed and theoretical EHVSRs at GIFH29 and KMMH14, and between observed and theoretical SRHSB at KMMH14 in the short period range, might imply that the assumed damping $(Q s=100)$ is not appropriate. This issue requires further scrutiny.

\section{Conclusions}

Based on the diffuse field approximation, FW $H / V$ is expressed with the imaginary parts of the Green's functions which incorporate the contributions of both body and surface (Rayleigh and Love) waves. SW $H / V$ is expressed with medium responses of Rayleigh and Love waves according to the Cauchy's contour integration method. For a given layered medium, the SW $H / V$ was almost the same as the FW $H / V$ in the short period range. It suggested that the contribution of surface waves trapped in shallow layers was overwhelmingly dominant compared with that of body waves. The largest peak of SW $H / V$ was caused by a local trough of fundamental or higher modes of vertical medium responses of Rayleigh waves. The predominant period of SW $H / V$ is close to but not identical to that of FW $H / V$ unless the impedance ratio is large enough. Therefore, the calculation of medium responses of Rayleigh and Love waves gave insight into the contribution of surface waves to fullwave, and the relative contribution between fundamental and higher modes of surface waves.

By adding a cap layer to the bottom of given layered medium, the SW $H / V$ of cap-layered medium agreed well with the FW $H / V$ of layered medium in the period range of engineering interest (e.g., 0.02-5.0 s). The depth of upper interface of the cap layer is about $(5-10) \lambda_{0}^{\mathrm{A}}$ (product of thickness-weighted average $\mathrm{S}$-wave velocity from surface to half-space of target layered medium and "apparent" predominant period of fundamental mode of target layered medium), and the velocity of the cap layer 

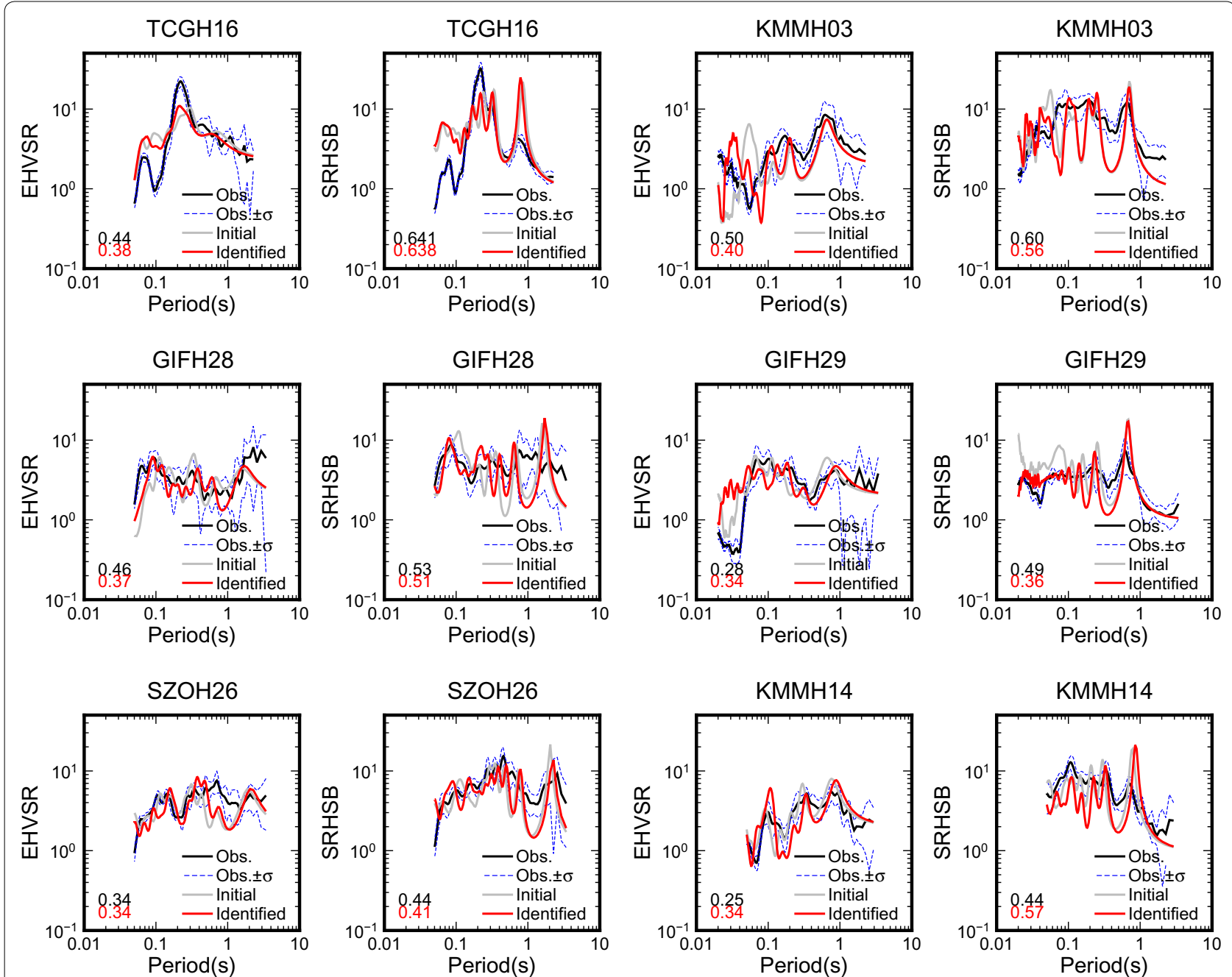

Fig. 10 Comparison between observed and theoretical EHVSRs, and between observed and theoretical SRHSBs at six KiK-net strong-motion stations. In the panels of EHVSRs, black curves denote the observed EHVSRs, dashed blue curves denote the observed EHVSRs with a standard deviation, gray curves denote the EHVSRs evaluated with the initial underground velocity structures, and red curves denote the EHVSRs evaluated with the identified underground velocity structures from surface to half-space without cap layer. In the panels of SRHSBs, black curves denote the observed SRHSBs, dashed blue curves denote the observed SRHSBs within one standard deviation, gray curves denote the SRHSBs evaluated with the initial underground velocity structures from surface to bottom of boreholes, and red curves denote the SRHSBs evaluated with the underground velocity structures from surface to bottom of boreholes identified from SW H/Vs. The numerical values in black represent the objective function Em between observed and theoretical EHVSRs (or SRHSBs) evaluated with initial underground velocity structures, and the numerical values in red represent the objective function Em between observed and theoretical EHVSRs (or SRHSBs) evaluated with identified underground velocity

structures

is about twice as large as the one in the half-space of given layered medium. Because the computation of surface waves was significantly fast, SW $H / V$ of cap-layered medium, as a simplified calculation of FW $H / V$ of layered medium without cap layer, was preferable to be applied to identify underground velocity structures.

We applied the SW $H / V$ of cap-layered medium to identify the underground velocity structures between surface and bottom of boreholes (about several hundred meters in depth) at some KiK-net stations where the FW $H / V$ s did not match with the observed $H / V$ s. We employed the identified underground velocity without the cap layer to evaluate the FW $H / V$. Good consistence between FW $H / V$ with $\mathrm{SW} H / V$ of the corresponding cap-layered medium was confirmed. It in turn suggested that SW $H / V$ could be regarded as a simplified calculation of FW $H / V$ to identify underground velocity structures. We further investigated the difference between SW $H / V$ of our approach and DSS-SW $H / V$ proposed on a different premise. Although the amplitude of DSS-SW $H / V$ was a bit smaller than our SW $H / V$, the similarity of these two SW $H / V \mathrm{~s}$ can be confirmed to some extent. 
Finally, we employed the identified underground velocity structures to evaluate earthquake motions of $H / V$ spectral ratios based on diffuse field approximation (EHVSR) and spectral ratios of horizontal motions between surface and bottom of boreholes (SRHSB). The good agreement between theoretical and observed EHVSR, and between theoretical and observed SRHSB, indicated that the underground velocity structures identified from $\mathrm{SW} H / V$ of cap-layered medium were well resolved by the new method.

This research implied verifying many details in order not to leave dead ends. The results are quite satisfactory because efficient, faster procedures for modeling and inversion of DFA $H / V$ were developed. Some fundamental issues and significant questions are still open, such as the effect of high damping in soft sediments, the sensitivity to the objective function, and the temporal variance of observations. We need to clarify these issues and open questions before our approach becomes a practical tool for field practitioners. This will require further scrutiny and we are committed in this noble endeavor.

\section{Additional files}

Additional file 1: Table S1. Underground velocity structure model at TCGH16. Table S2. Underground velocity structure model at KMMH03. Table S3. Underground velocity structure model at GIFH28.

Table S4. Underground velocity structure model at GIFH29.

Table S5. Underground velocity structure model at $\mathrm{SZOH} 26$.

Table S6. Underground velocity structure model at $\mathrm{KMMH} 14$.

Additional file 2: Table S1. Small events at TCGH16. Table S2. Small events at KMMH03. Table S3. Small events at GIFH28. Table S4. Small events at GIFH29. Table S5. Small events at SZOH26. Table S6. Small events at KMMH14.

\section{Abbreviations}

DFA: diffuse field approximation; $H / V$ : microtremor $H / V$ spectral ratio; FW: full-wave; SW: surface waves; DSS-SW: surface waves based on distributed surface sources assumption; EHVSR: earthquake motions of H/V spectral ratio; SRHSB: spectral ratio of horizontal motions between surface and bottom of borehole; $\mathrm{Tp}_{0}$ : predominant period of fundamental mode; $\lambda_{0}$ : wavelength of fundamental mode; $T p_{0}^{A}$ : "apparent" predominant period of fundamental mode; $\lambda_{0}^{A}$ : "apparent" wavelength of fundamental mode; $V_{s}$ : S-wave velocity; $V_{p}$ : P-wave velocity.

\section{Authors' contributions}

HW designed this study and drafted the manuscript. KM and HW conducted the microtremor measurement at strong-motion stations. Kl participated in all discussions throughout the study. FS participated in the discussions and interpretation. All authors read and approved the final manuscript.

\section{Author details}

${ }^{1}$ Earthquake Engineering Group, Geo-Research Institute, 2-1-2, Otemae, Chuo-ku, Osaka, Japan. ${ }^{2}$ Disaster Prevention Research Center, Aichi Institute of Technology, Yachigusa 1247, Yakusa-cho, Toyota, Japan. ${ }^{3}$ Instituto de Ingeniería, Universidad Nacional Autónoma de México, C.U., Coyoacán 04510, CDMX, Mexico.

\section{Acknowledgements}

The authors wholeheartedly thank two anonymous reviewers and the Guest Editor PY Bard, for their detailed review comments and most valuable editing suggestions of the admittedly rough, initial manuscript. Special thanks to the National Research Institute for Earth Science and Disaster Resilience (NIED) for providing PS logging data and strong-motion records at KiK-net stations. We thank E. Plata, G. Sánchez, and their team of the Unidad de Servicios de Información (USI) of the Institute of Engineering - UNAM, for locating useful references. This work was supported in part by the Grants-in-Aids for Scientific Research (B) (P.I.: Hidenori Kawabe, Grant Number: 16H03144) from the Ministry of Education, Culture, Sports, Science and Technology of Japan, by AXA Research Fund and by DGAPA-UNAM under Project IN100917. Figures are plotted with Generic Mapping Tools (GMT) (Wessel et al. 2013).

\section{Competing interests}

The authors declare that they have no competing interests.

\section{Availability of data and materials}

Strong-motion records at studied KiK-net stations can be downloaded from the website "http://www.kyoshin.bosai.go.jp/kyoshin/" after a free registration. PS logging data (thickness, P-wave velocity, S-wave velocity in each layer) are available from the Web site "http://www.kyoshin.bosai.go.jp/kyoshin/db/ index.html?all".

\section{Ethics approval and consent to participate} Not applicable.

\section{Publisher's Note}

Springer Nature remains neutral with regard to jurisdictional claims in published maps and institutional affiliations.

Received: 21 December 2016 Accepted: 15 November 2017

Published online: 04 December 2017

\section{References}

Aki K (1957) Space and time spectra of stationary stochastic waves with special reference to microtremors. Bull Earthq Res Inst 35:415-456

Albarello D, Lunedei E (2013) Combining horizontal ambient vibration components for H/N spectral ratio estimates. Geophys J Int 194:936-951

Arai H, Tokimatsu K (2004) S-wave velocity profiling by inversion of microtremor H/N spectrum. Bull Seismol Soc Am 94(1):53-63

Arai H, Tokimatsu K (2005) S-wave velocity profiling by joint inversion of microtremor dispersion curve and horizontal-to-vertical $(\mathrm{H} / \mathrm{N})$ spectrum. Bull Seismol Soc Am 95(5):1766-1778

Baan M (2009) The origin of SH-wave resonance frequencies in sedimentary layers. Geophys J Int 178:1587-1596

Bonnefoy-Claudet S, Cornou C, Bard PY, Cotton F, Moczo P, Kristek J, Fäh D (2006a) H/V ratio: a tool for site effects evaluation: results from $1 D$ noise simulations. Geophys J Int 167(2):827-837

Bonnefoy-Claudet S, Cotton F, Bard PY (2006b) The nature of noise wavefield and its applications for site effects studies: a literature review. Earth Sci Rev 79:205-227

Bonnefoy-Claudet S, Köhler A, Cornou C, Wathelet M, Bard PY (2008) Effects of Love waves on microtremor H/N ratio. Bull Seismol Soc Am 98(1):288-300

Ducellier A, Kawase H, Matsushima S (2013) Validation of a new velocity structure inversion method based on horizontal-to-vertical (H/N) spectral ratios of earthquake motions in the Tohoku Area, Japan. Bull Seismol Soc Am 103(2A):958-970

Fäh D, Kind F, Giardini D (2001) A theoretical investigation of average H/N ratios. Geophys J Int 145:535-549

García-Jerez A, Luzón F, Albarello D, Lunedei E, Santoyo MA, Margerin L, Sánchez-Sesma FJ (2012) Comparison between ambient vibrations HN obtained from the diffuse field and the distributed surface sources models. In: Proceedings of 15th world conference on earthquake engineering, Lisbon, Portugal 
García-Jerez A, Luzón F, Sánchez-Sesma FJ, Lunedei E, Albarello D, Santoyo MA, Almendros J (2013) Diffuse elastic wavefield within a simple crustal model. Some consequences for low and high frequencies. J Geophys Res Solid Earth 118(10):5577-5595

García-Jerez A, Piña-Flores J, Sánchez-Sesma FJ, Luzón F, Perton M (2016) A computer code for forward calculation and inversion of the H/N spectral ratio under the diffuse field assumption. Comput Geosci 97:67-78. https://doi.org/10.1016/j.cageo.2016.06.016

Harkrider DG (1964) Surface waves in multilayered elastic media, I. Rayleigh and Love waves from buried sources in multilayered elastic half-space. Bull Seismol Soc Am 54(2):627-679

Harvey DJ (1981) Seismogram synthesis using normal mode superposition: the locked mode approximation. Geophys J R Astron Soc 66:37-69

Haskell NA (1960) Crustal reflection of plane SH waves. J Geophys Res 65(12):4147-4150

Hennino R, Tregoures N, Shapiro N, Margerin L, Campillo M, Tiggelen van Weaver R (2001) Observation of equipartition of seismic waves. Phys Rev Lett 86:3447-3450

Kawase H, Sánchez-Sesma FJ, Matsushima S (2011) The optimal use of horizontal-to-vertical spectral ratios of earthquake motions for velocity inversions based on diffuse-field theory for plane waves. Bull Seismol Soc Am 101(5):2001-2014

Kawase H, Matsushima S, Satoh T, Sánchez-Sesma FJ (2015) Applicability of theoretical horizontal-to-vertical ratio of microtremors based on the diffuse field concept to previously observed data. Bull Seismol Soc Am 105(6):3092-3103

Konno K, Ohmachi T (1998) Ground-motion characteristics estimated from spectral ratio between horizontal and vertical components of microtremor. Bull Seismol Soc Am 88:228-241

Lachet C, Bard PY (1994) Numerical and theoretical investigations on the possibilities and limitations of Nakamura's technique. J Phys Earth 42(5):377-397

Lawrence JF, Prieto GA (2011) Attenuation tomography of the western United States from ambient seismic noise. J Geophys Res Solid Earth 116:B06302

Lermo J, Chávez-García FJ (1993) Site effects evaluation using spectral ratios with only one station. Bull Seismol Soc Am 83(5):1574-1594

Lin CM, Wen KL, Kuo CH, Lin CY (2014) S-wave velocity model of Taipei Basin. In: The 5th Asia conference on earthquake engineering, October 16-18, Taipei

Lontsi AM, Sánchez-Sesma FJ, Molina-Villegas JC, Ohrnberger M, Krüger F (2015) Full microtremor H/N(z, f) inversion for shallow subsurface characterization. Geophys J Int 202:298-312

Lunedei E, Albarello D (2009) On the seismic noise wavefield in a weakly dissipative layered Earth. Geophys J Int 177:1001-11014

Lunedei E, Albarello D (2010) Theoretical HVSR curves from full wavefield modelling of ambient vibrations in a weakly dissipative layered Earth. Geophys J Int 181:1093-1108

Lunedei E, Albarello D (2015) Horizontal-to-vertical spectral ratios from a full-wavefield model of ambient vibrations generated by a distribution of spatially correlated surface sources. Geophys J Int 201:1140-1153

Margerin L (2009) Generalized eigenfunctions of layered elastic media and application to diffuse fields. J Acoust Soc Am 125(1):164-174

Mulargia F (2012) The seismic noise wavefield is not diffuse. J Acoust Soc Am 131(4):2853-2858

Nakamura Y (1989) A method for dynamic characteristics estimation of subsurface using microtremor on ground surface. Railw Tech Res Inst Q Rep 30(1):25-33

Nakamura Y (2000) Clear identification of fundamental idea of Nakamura's technique and its applications. In: Proceedings 12th world conference on earthquake engineering, Auckland, New Zealand

Nolet G, Sleeman R, Nijhof V, Kennett BLN (1989) Synthetic reflection seismograms in three dimensions by a locked-mode approximation. Geophysics 54(3):350-368

Okada H (2003) The microtremor survey method. Geophysical monograph series, No. 12 (DV Fitterman and MW Asten Editors), Society of Exploration Geophysicists, Tulsa, Oklahorma, USA

Parolai S, Picozzi M, Richwalski SM, Milkereit C (2005) Joint inversion of phase velocity dispersion and $\mathrm{H} / \mathrm{N}$ ratio curves from seismic noise recordings using a genetic algorithm, considering higher modes. Geophys Res Lett 32:L01303. https://doi.org/10.1029/2004GL021115
Picozzi M, Parolai S, Richwalski SM (2005) Joint inversion of H/N ratios and dispersion curves from seismic noise: estimating the S-wave velocity of bedrock. Geophys Res Lett 32:L11308. https://doi.org/10.1029/2005GL022878

Piña-Flores J, Perton M, García-Jerez A, Carmona E, Luzón F, Molina-Villegas JC, Sánchez-Sesma FJ (2017) The inversion of spectral ratio H/V in a layered system using the diffuse field assumption (DFA). Geophys J Int 208:577-588

Sabra KG, Gerstoft P, Roux P, Kuperman WA, Fehler MC (2005) Extracting timedomain Green's function estimates from ambient seismic noise. Geophys Res Lett 32:L03310. https://doi.org/10.1029/2004GL021862

Saguchi K, Masaki K, Irikura K (2009) Estimation of strong motions on free rock surface-identification of soil structures and strong motions on free rock surface in Kashiwazaki-Kariwa nuclear power plant during the $2007 \mathrm{Nii}-$ gataken Chuetsu-oki earthquake. J Struct Constr Eng AlJ 74(639):831-839 (in Japanese with English abstract)

Saito M, Kabasawa H (1993) Computations of reflectivity and surface wave dispersion curves for layered media II. Rayleigh Wave Calculations. BUTSURITANSA 46(4):283-298 (in Japanese with English abstract)

Sánchez-Sesma FJ, Campillo M (2006) Retrieval of the Green's function from cross-correlation: the canonical elastic problem. Bull Seismol Soc Am 96:1182-1191

Sánchez-Sesma FJ, Pérez-Ruiz JA, Luzón F, Campillo M, Rodríguez-Castellanos A (2008) Diffuse fields in dynamic elasticity. Wave Motion 45:641-654

Sánchez-Sesma FJ, Rodríguez M, Iturrarán-Viveros U, Luzón F, Campillo M, Margerin L, García-Jerez A, Suárez M, Santoyo MA, Rodríguez-Castellanos A (2011) A theory for microtremor HN spectral ratio: application for a layered medium. Geophys J Int 186(1):221-225

Satoh T (2006) Inversion of Qs of deep sediments from surface-to-borehole spectral ratios considering obliquely incident SH and SV-waves. Bull Seismol Soc Am 96:943-956

Shapiro NM, Campillo M (2004) Emergence of broadband Rayleigh waves from correlations of the ambient seismic noise. Geophys Res Lett 31:L07614. https://doi.org/10.1029/2004GL 019491

Shapiro NM, Campillo M, Margerin L, Singh SK, Kostoglodov V, Pacheco J (2000) The energy partitioning and the diffusive character of the seismic coda. Bull Seismol Soc Am 90:655-665

Shapiro NM, Campillo M, Stehly L, Ritzwoller MH (2005) High-resolution surface-wave tomography from ambient seismic noise. Science 307(5715):1615-1618

Snieder R, Wapenaar K, Wegler U (2007) Unified Green's function retrieval by crosscorrelation; connection with energy principles. Phys Rev $E$ 75:036103-1-03610303610314

Tamura S (1996) Comparison of body and Rayleigh wave displacements generated by a vertical force on a layered elastic medium. In: Proceedings 11 th world conference on earthquake engineering

Thompson EM, Baise LG, Tanaka Y, Kayen RE (2012) A taxonomy of site response complexity. Soil Dyn Earthq Eng 41:32-43

Tokimatsu K, Tamura S (1995) Contribution of Rayleigh and body waves to displacement induced by a vertical point force on a layered elastic half-space. J Struct Constr Eng 476:95-101 (in Japanese with English abstract)

Wang X, Kurahashi S, Wu H, Si HJ, Ma Q, Dang J, Tao DW, Feng JW, Irikura K (2017) Building damage concentrated in Longtoushan town during the 2014 Ms6.5 Ludian Earthquake, Yunnan, China: examination of cause and implications based on ground motion and vulnerability analyses. J Seismol. https://doi.org/10.1007/s10950-017-9659-z

Weaver RL (1982) On diffuse waves in solid media. J Acoust Soc Am 71:1608-1609

Weaver RL, Lobkis Ol (2001) On the emergence of the Green's function in the correlations of a diffuse field. J Acoust Soc Am 110:3011-3017

Wessel P, Smith WH, Scharroo R, Luis JF, Wobbe F (2013) Generic mapping tools: improved version released. Eos Trans Am Geophys Union 94:409-410

Wu H, Masaki K, Irikura K, Saguchi K, Kurahashi S, Wang X (2012) Relationship between building damage ratios and ground motion characteristics during the 2011 Tohoku Earthquake. J Nat Disaster Sci 34(1):59-78

Wu H, Masaki K, Irikura K, Saguchi K, Kurahashi S (2016) Empirical fragility curves of buildings in northern Miyagi prefecture during the 2011 off the Pacific Coast of Tohoku Earthquake. J Disaster Res 11(6):1253-1270 